\title{
Practical Aspects for the Integration of 5G Networks and IoT Applications in Smart Cities Environments
}

\author{
Daniel Minoli (iD) and Benedict Occhiogrosso \\ DVI Communications, Inc. New York, NY, USA \\ Correspondence should be addressed to Daniel Minoli; daniel.minoli@dvicomm.com
}

Received 1 April 2019; Accepted 26 May 2019; Published 5 August 2019

Guest Editor: Xi Chen

Copyright (c) 2019 Daniel Minoli and Benedict Occhiogrosso. This is an open access article distributed under the Creative Commons Attribution License, which permits unrestricted use, distribution, and reproduction in any medium, provided the original work is properly cited.

\begin{abstract}
Both 5G cellular and IoT technologies are expected to see widespread deployment in the next few years. At the practical level, 5G will see initial deployments in urban areas. This is perhaps fortuitous from an IoT perspective, since many "mainstream" applications of IoT will support Smart Cities, Smart Campuses and Smart Buildings. Bandwidth demand for a number of Smart City applications is the main driver for enhanced mobile broadband (eMBB)-based 5G services in general, and new-generation 5G IoT applications, in particular. In turn, the use of the millimeter wave spectrum is required to enable $5 \mathrm{G}$ cellular technologies to support high data rates. Millimeter wave solutions, however, impose a requirement for small cells. Generally, an implementer tries to use one or a small handful of IoT technologies; preferably, and for managerial simplicity, the implementer would want to use a cellular/5G IoT technology for all nodes, whether indoors or outdoors, instead of a heterogenous mix of various IoT technologies that have evolved over the years. This overview paper discusses a number of practical issues related to 5G-based IoT applications, particularly in Smart City environments, including the need for small cells, the transmission issues at millimeter wave frequencies, building penetration issues, the need for Distributed Antenna Systems, and the near term introduction of pre-5G IoT technologies such as NB-IoT and LTE-M, these being possible proxies for the commercial deployment and acceptance of 5G IoT.
\end{abstract}

\section{Background}

As the second decade of the $21^{\text {st }}$ century comes to a close, we are witnessing an expansion of urban ecosystems, as populations continue to sustain the transition from rural and some suburban areas into large urban areas, driven by economic opportunities, demographic shifts and generational preferences. Seventy percent of the human population is expected to live in cities by the year 2050, and there already are more than 400 cities with over one million inhabitants [1]. Societal movement of people is a basic human existence dynamic and is one of the key mechanisms that drives the growth of cities. Yet, especially in the Western World, cities often have aging infrastructure, including roads, bridges, tunnels, rail yards, and power distribution plants. It follows that new technological solutions are needed to optimize the increasingly-scarce infrastructure resources, especially given the population growth and the limited financial resources available to most cities and municipalities. When cities deploy state-of-the-art Information and Communication Technologies (ICT) on a large-scale, including Internet of Things (IoT) technologies, they are referred to as being "Smart Cities".

Livability, infrastructure management, asset management, traffic transportation and mobility, logistics, electric power and other utilities, and physical security are the key aspects of a city's operation. IoT technologies offer the opportunity to improve resource management of many assets related to city life and urban Quality of Life (QoL), including Intelligent Transportation Systems (vehicular automation and traffic control), energy consumption, the flow of goods, smart buildings, space/occupancy management (indoors and outdoors), pollution monitoring (for example from automobile traffic, factories, incinerators, crematoria), resource monitoring and sensing, immersive services (including wearables and crowdsensing), physical security, sustainability, and the greening of the environment. Smart City IoT applications cover indoor and outdoor applications; they also span 


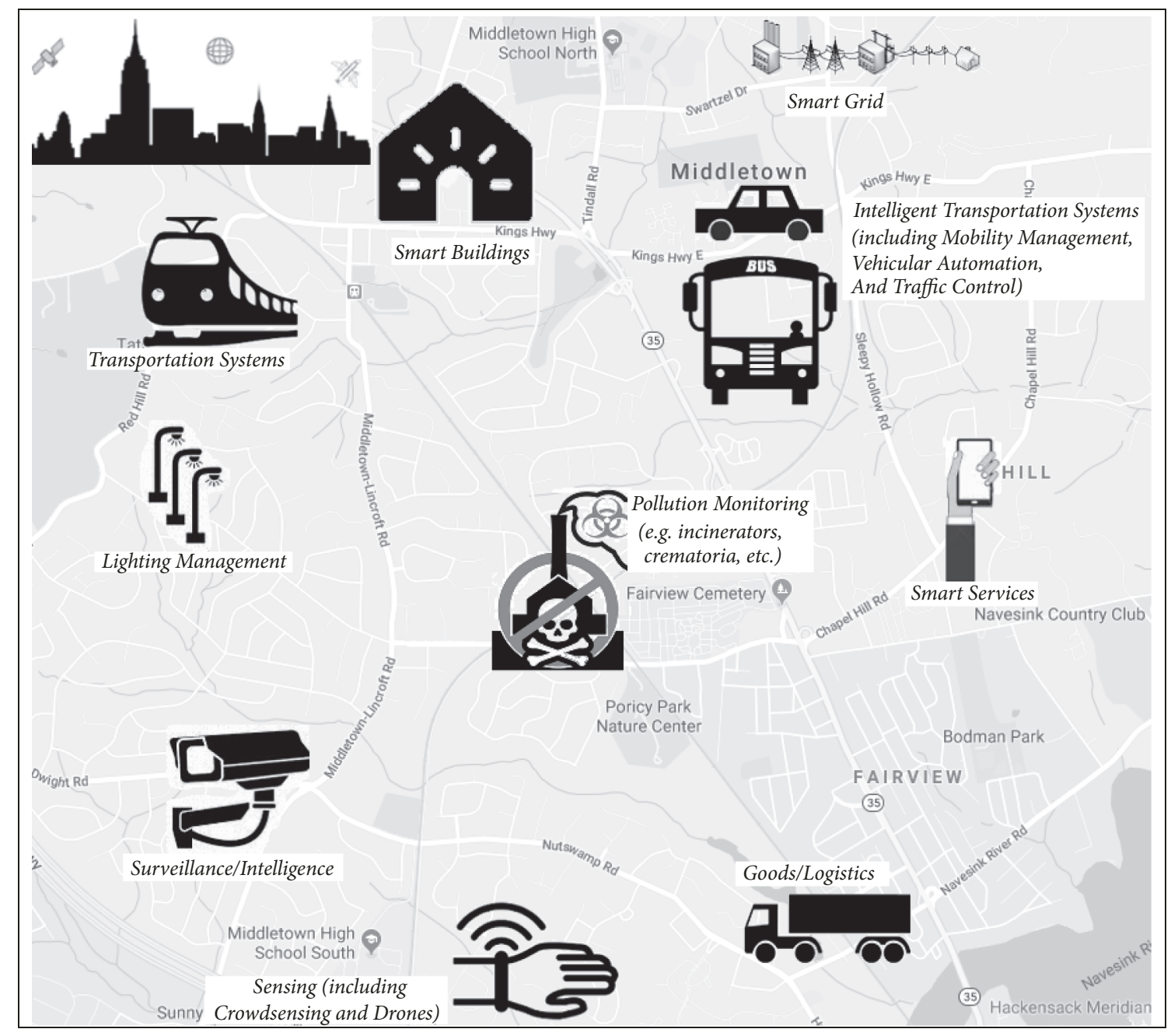

FIGURE 1: Illustrative example of Smart City resources that can benefit from IoT in general and 5G cellular in particular.

stationary and mobile end-nodes and sensors. There is an extensive body of literature on this topic; some references of interest include but are certainly not limited to [2-12]. Up to the present, the IoT has been mostly utilized for supporting a large population of relatively low-bandwidth sensing devices, and where the sensing devices are typically deployed in stationary locations (e.g., electric meters, building management systems, meteorological weather stations). However, video-oriented applications that require streams ranging up to Ultra-High Definition resolution are emerging (e.g., surveillance, physical security). In the evolving IoT environment, the endpoint devices include environmental and situational sensors, vehicles, wearables, drones, robots, and Virtual Reality gear. In some applications IoT actuators are also utilized to control the physical ecosystem in response to a sensed set of data or some analytical calculation - for example, changing the barriers and signs on a road to reverse traffic lanes during the day; or, changing the parameters of a pump to control water or sewer flows. Figure 1 depicts graphically some of the common Smart City applications.

While a large number of definitions and descriptions of the IoT exist, this is one definitional/descriptive quote from the author's previous work, which we utilize here: "The basic concept of the IoT is to enable objects of all kinds to have sensing, actuating, and communication capabilities, so that locally-intrinsic or extrinsic data can be collected, processed, transmitted, concentrated, and analyzed for either cyber-physical goals at the collection point (or perhaps along the way), or for process/environment/systems analytics (of a predictive or historical nature) at a processing center, often "in the cloud". Applications range from infrastructure and criticalinfrastructure support (for example smart grid, Smart City, smart building, and transportation), to end-user applications such as e-health, crowdsensing, and further along, to a multitude of other applications where only the imagination is the limit" [13-27]. According to the Global System for Mobile Communications Association (GSMA), between 2018 and 2025, the number of global IoT connections will triple to 25 billion, while global IoT revenue will quadruple to $\$ 1.1$ trillion [28]; others offer higher numbers (e.g., according to Statistica there will be about 80 billion IoT devices worldwide in 2025 [29]).

$5 \mathrm{G}$ ( $5^{\text {th }}$ Generation) is the term for the next-generation cellular/wireless service provider network that aims at 
delivering higher data rates -- 100 times faster data speeds than the current $4 \mathrm{G}$ Long Term Evolution (LTE) technology -- lower latency, and highly-reliable connectivity. In a sense, it is an evolution of the previous generations of cellular technology.

Smart Cities do not depend on any unique or specific IoT technology per se, but include a panoply of IoT technologies, such as mission-specific sensors, appropriate networks, and function-and-use-efficient analytics, these often in the cloud. Wireless connectivity plays an important role in the utility of this technology, especially at the geographic scope of a large or even medium-size city. For practical reasons wireless is also important in Smart Campus and Smart Building applications. Table 1 identifies a number of Smart City challenges and needs, possible IoT-based solutions, wireless requirements, and the applicability of $5 \mathrm{G}$ solutions. $5 \mathrm{G}$ IoT is licensed cellular IoT. In this table "low bandwidth" equates to $200 \mathrm{kbps}$ or less, "medium bandwidth" equates to 200 kbps to $2 \mathrm{Mbps}$, and "high bandwidth" equates to more than 2 Mbps. Some IoT applications entail periodic "batch" communication while other applications require real-time communication; in the table "low latency" means real-time and "medium latency" means 1-to-5 seconds. Table 2 provides a snapshot of key wireless technologies that are applicable to the IoT environment. A number of wireless technologies are available, each with its specific applicability and functionality. The direct use of traditional cellular services (e.g., 4G/LTE networks) is not optimal for IoT applications, both for cost and nodal power-consumption reasons. Furthermore, these services are not ideal for a number of IoT applications where a small amount of data is transmitted infrequently (e.g., electric, gas, or water meters for reading). Node density is also an issue. Cellular IoT solutions endeavor to address low-power, low data rate requirements. Several iterations and alternatives solutions have emerged in recent years (e.g., Cat1/Rel 8, Cat 0/Rel 12, Cat-M/Rel 13, EC-GSM, and NBIoT/Rel 13). The 5G IoT system is the next evolutionary step, perhaps also affording some simplification and technology homogeneity.

Figure 2 depicts the pre-5G and the $5 \mathrm{G}$ IoT connectivity ecosystem, which is further elaborated in the rest of this paper. The figure illustrates a typical case of Wi-Fi (inbuilding) aggregation of sensor data for a handoff to the cloud over a traditional router; it illustrates the use of Low Power Wide Area Network (LPWAN) overlay technologies such as LoRa and Sigfox; it shows the use of pre-5G IoT technologies; and then illustrates the use of 5G IoT in a native mode, or in a more realistic Distributed Antenna System (DAS)-assisted mode.

This review, position and assessment paper provides an overview of salient $5 \mathrm{G}$ features and then discusses some practical design issues applicable to the IoT. A lot of the important 5G IoT information is included in the figures and tables. This paper is not intended to be a full $5 \mathrm{G}$ overview per se, nor a discussion of IoT, for both of which there are many references (e.g., [30-34] for 5G, and close to one hundred books on the IoT topic alone).

\section{5G Concepts and Technology}

$5 \mathrm{G}$ cellular networks are now starting to be deployed around the world, as the underlying standards and the system-wide technology become more mature (the term "International Mobile Telecommunications-2020 [IMT-2020]" is also used by the standards bodies.) Industry observers predict that societal developments will lead to changes in the way communication systems are used and that these developments will, in turn, lead to a significant increase in mobile and wireless traffic volume; such traffic volume is expected to increase a thousandfold over the next decade. Observations such as this one are common in the literature positioning the technology: "Unlike previous generations of mobile networks, the fifth generation (5G) technology is expected to fundamentally transform the role that telecommunications technology plays in the society" [34].

The $5 \mathrm{G}$ system expands the $4 \mathrm{G}$ environment by adding New Radio (NR) capabilities, but doing so in such a manner that LTE and NR can evolve in complementary ways. As it might be envisioned, a $5 \mathrm{G}$ system entails devices connected to a $5 \mathrm{G}$ access network, which in turn is connected to a $5 \mathrm{G}$ core network. The 5G access network may include 3GPP (3rd Generation Partnership Project) radio base stations and/or a non-3GPP access network. The 5G core network offers major improvements compared with a $4 \mathrm{G}$ system in the area of network slicing and service-based architectures (SBAs); in particular, the core is designed to support cloud implementation and the IoT. 5G systems subsume important $4 \mathrm{G}$ system concepts such as the energy saving capabilities of narrowband IoT (NB-IoT) radios, secure low latency small data transmission for low-power devices -- low latency is a requirement for making autonomous vehicles safe -and devices using energy-preserving dormant states when possible. Network slicing allows service providers to deliver "Network as a Service (NaaS)" to large/institutional users affording them the flexibility to manage their own services and devices on the $5 \mathrm{G}$ provider's network.

Applications driving wireless traffic include but are not limited to on-demand mobile information and highresolution entertainment, augmented reality, virtual reality and immersive services, e-health, and ubiquitous IoT rollouts. While 5G technology could still take several distinct service directions, it appears at this juncture that the view favoring a super-fast mobile network, where densely-clustered small cells provide contiguous urban coverage to mobile as well as stationary users, is the approach envisioned by the standards development bodies and by the implementers. It should be noted that in the U.S., upwards of 55 percent of residential users now utilize cellular-services-only at home in place of a landline, and about 30 percent of residential users utilize both, with the trend favoring an eventual transition to the former. Therefore, the evolving $5 \mathrm{G}$ systems will have to properly support this growing segment of the market. A goal of $5 \mathrm{G}$ networks is to be five times as fast as compared to the highest current speed of existing $4 \mathrm{G}$ networks, with download speeds as high as $5 \mathrm{Gbps}-4 \mathrm{G}$ offering only up to a maximum of 1 Gbps. Deployment of $5 \mathrm{G}$ networks started in 2018 in some advanced countries, although further developments on fundamentals will continue; naturally, the current 


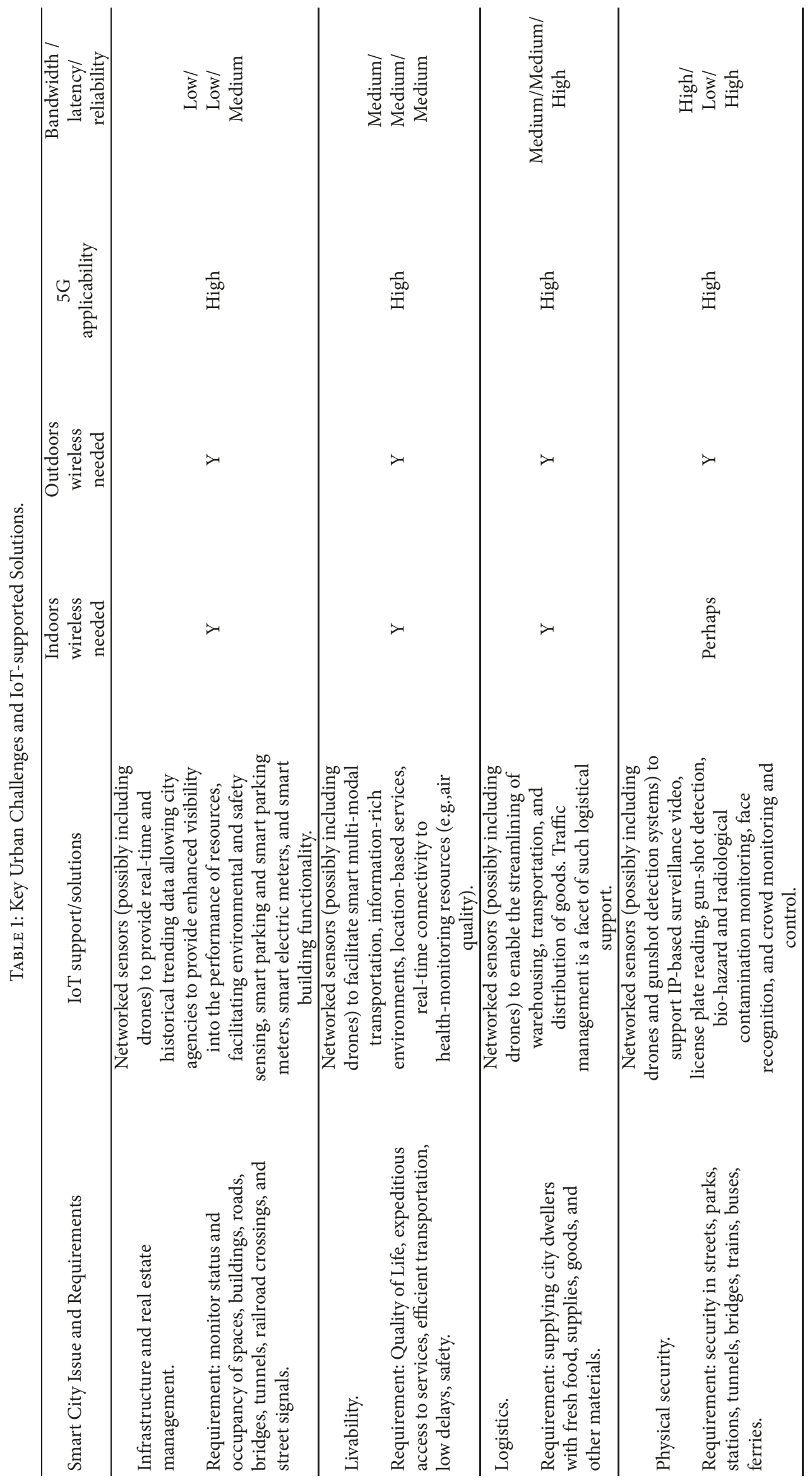




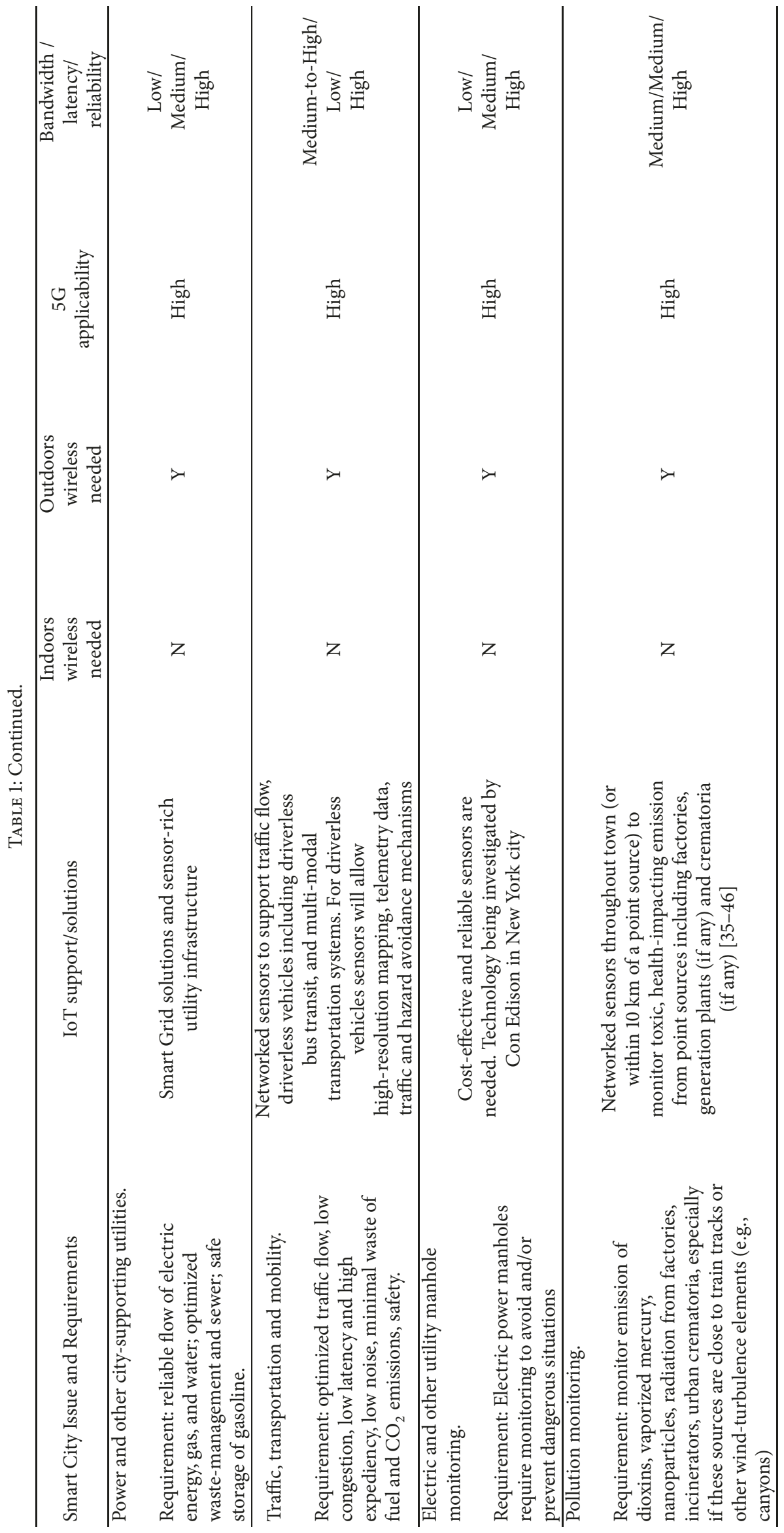




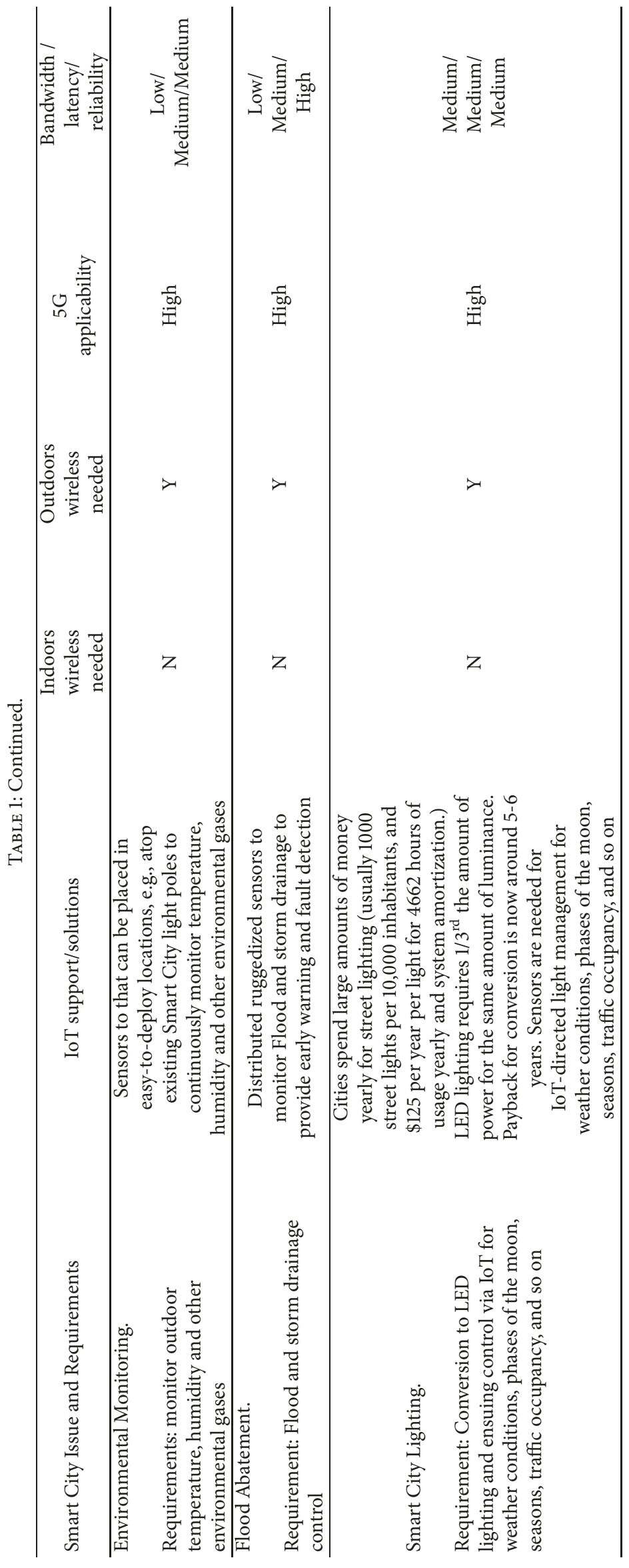




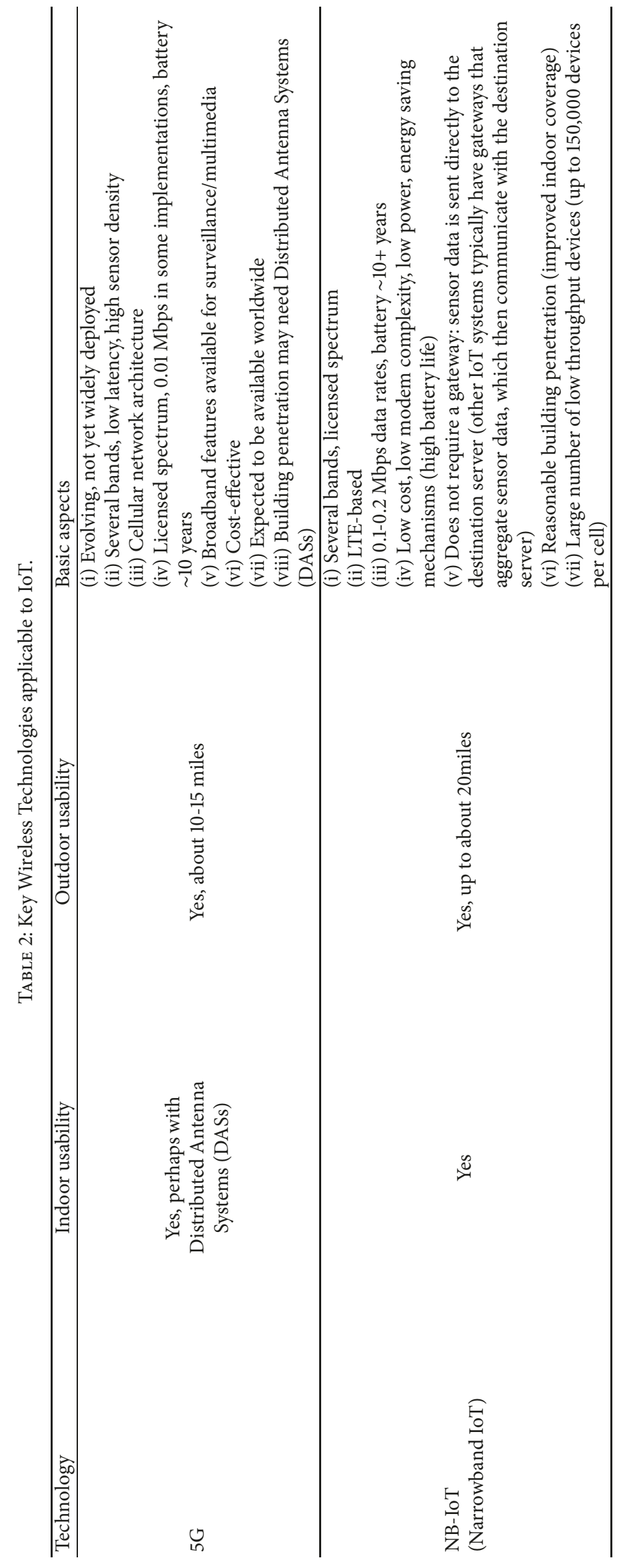




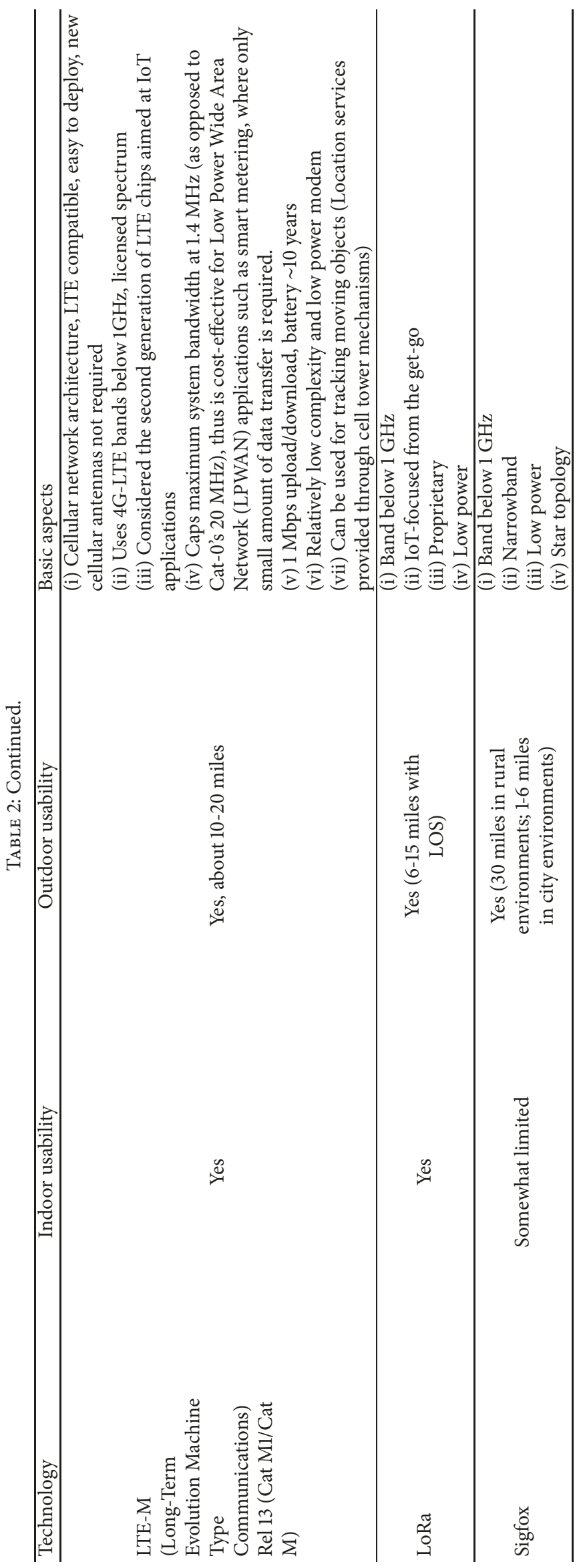




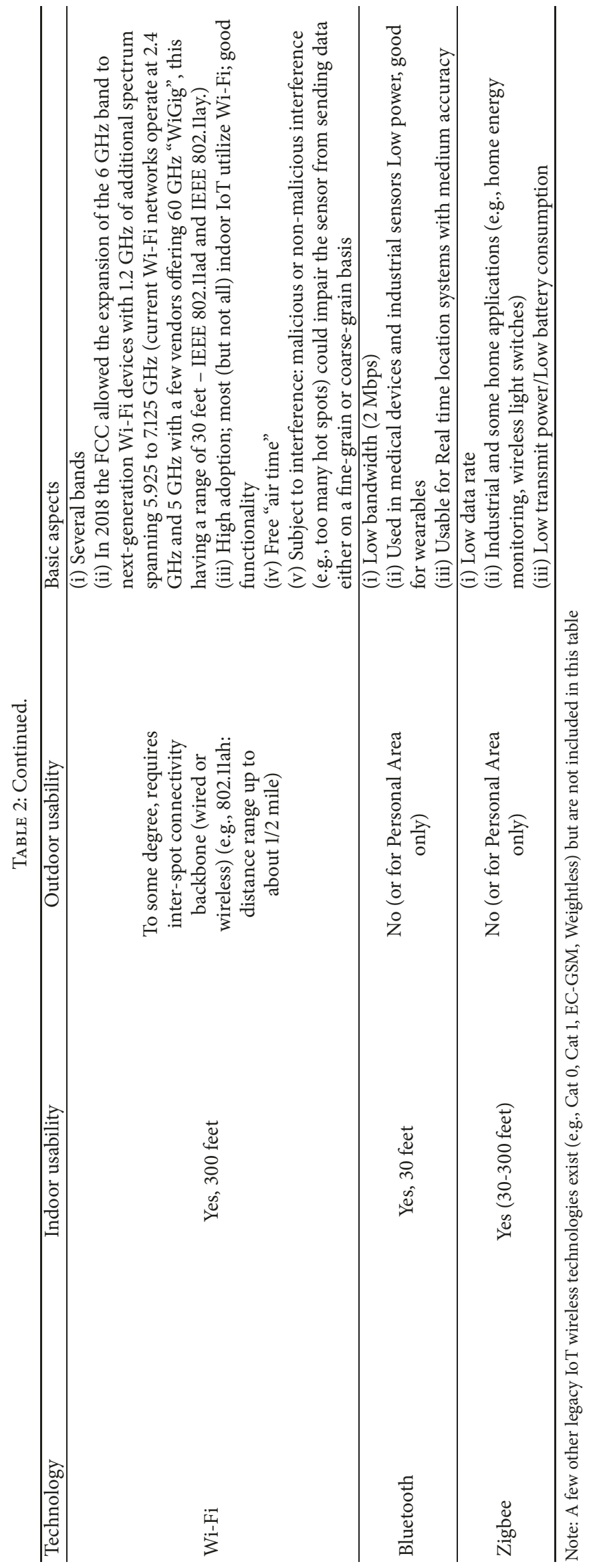




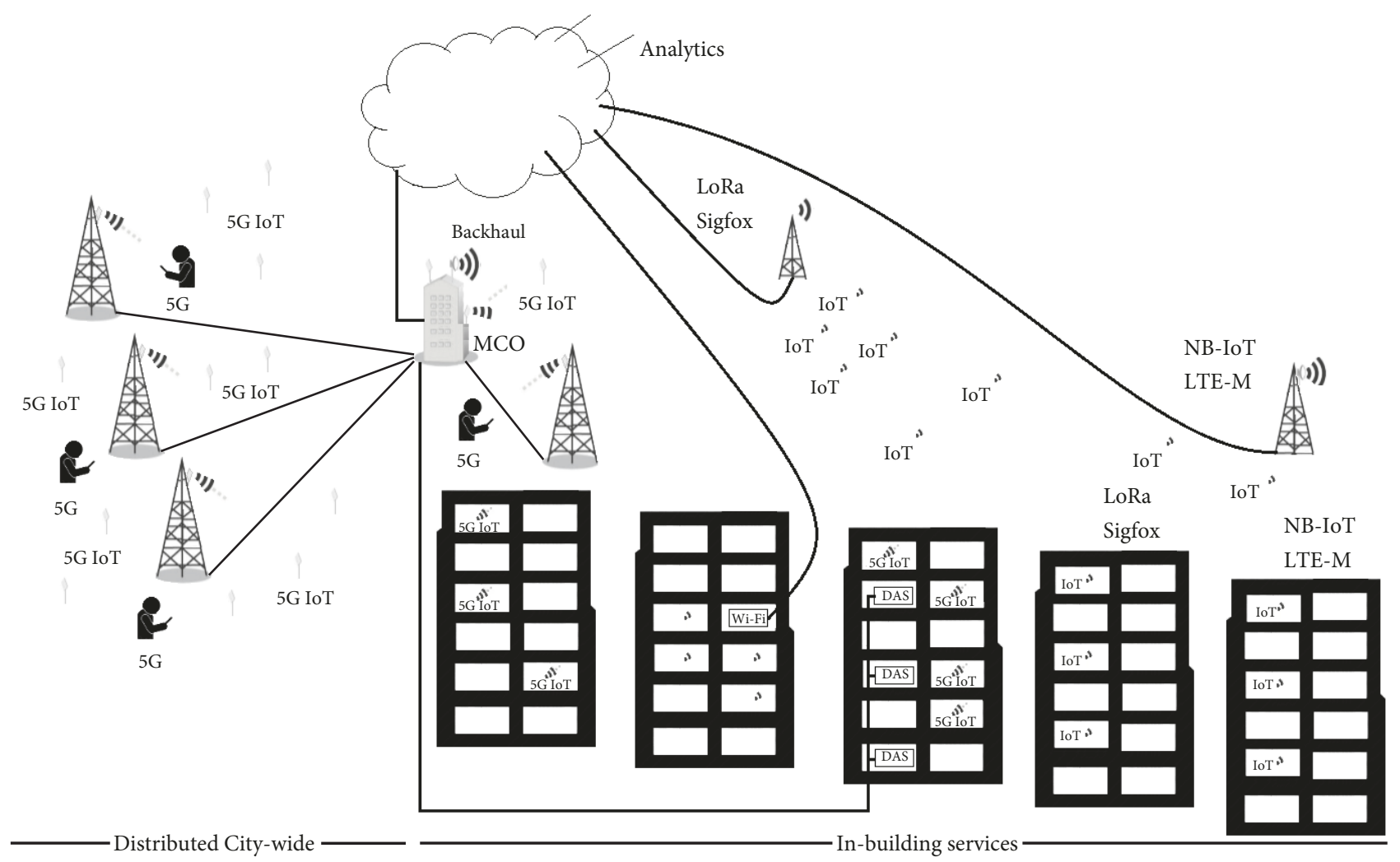

FIgURE 2: The pre-5G and the 5G IoT connectivity ecosystem.

4G/LTE and 5G are expected to coexist for many years. However, it is fair to say that like many other technologies before $5 \mathrm{G}$, this technology is probably going through a "hypecycle", where a technology is supposed to be "all things to all people" and be the "be-all-and-end-all technology"; both claims will be abrogated in time. Proponents argue that $5 \mathrm{G}$ will "maximize the satisfaction of end-users by providing immersiveness, intelligence, omnipresence, and autonomy”.

2.1. 5 G Standardization and Use Cases. Standardization work for $5 \mathrm{G}$ systems has been undertaken by several international bodies with the goal of achieving one unified global standard. Many well-known research centers, universities, standards bodies, carriers and technology providers have been involved in advancing the development of the technology for a 2020 rollout, including the Internet Engineering Task Force (IETF), the Open Network Automation Platform (ONAP), the GSMA, and the European Telecommunications Standards Institute Network Function Virtualization (ETSI NFV). In particular, work on $5 \mathrm{G}$ requirements, services and technical specifications has been undertaken in the past few years by three key entities: (i) International Telecommunication Union-Radio Communication Sector (ITU-R) [30], (ii) Next Generation Mobile Networks (NGMN) Alliance [31], and (iii) the 3rd Generation Partnership Project (3GPP) [32]. The ITU-R has assessed usage scenarios in three classes: ultrareliable and low-latency communications (URLLC), massive machine-type communications (mMTC), and enhanced mobile broadband (eMBB). eMBB is probably the earliest class of services being broadly supported and implemented. Key performance indicators are identified for each of these classes, such as spectrum efficiency, area traffic capacity, connection density, user-experienced data rate, peak data rate, and latency, among others. The ability to efficiently handle device mobility is also critical. Some examples of eMBB use cases include Non-SIM devices, smart phones, home/enterprise/venues applications, UHD (4K and $8 \mathrm{~K}$ ) broadcast, and virtual reality/augmented reality. mMTC use cases include smart buildings, logistics, tracking, fleet management and smart meters. URLLC cases include traffic safety and control, remote surgery, and industrial control. 5G systems are expected to support:

(i) Tight latency, availability, and reliability requirements to facilitate applications related to video delivery, healthcare, surveillance and physical security, logistics, automotive locomotion, and mission-critical control, among others, particularly in an IoT context;

(ii) A panoply of data rates, up to multiple Gbps, and tens of Mbps to facilitate existing and evolving applications, particularly in an IoT context;

(iii) Network scalability and cost-effectiveness to support both clustered users with very high data rate requirements as well a large number of distributed devices with low complexity and limited power resources, particularly in an IoT context, where, as noted, a rapid increase in the number of connected devices is anticipated; and, 
TABLE 3: Radio interface goals as defined in IMT-2020.

(i) MR for downlink peak data rate is $20 \mathrm{Gbps}$

(ii) MR for uplink peak data rate is $10 \mathrm{Gbps}$

(iii) Target downlink "user experienced data rate" is $100 \mathrm{Mbps}$

(iv) Target uplink "user experienced data rate" is $50 \mathrm{Mbps}$

(v) Downlink peak spectral efficiency is $30 \mathrm{bps} / \mathrm{Hz}$

(vi) Uplink peak spectral efficiency is $15 \mathrm{bps} / \mathrm{Hz}$

(vii) MR for user plane latency for eMBB is $4 \mathrm{~ms}$

(viii) MR for user plane latency for URLLC is $1 \mathrm{~ms}$

(ix) MR for control plane latency is $20 \mathrm{~ms}$ (a lower control plane latency of around $10 \mathrm{~ms}$ is encouraged)

(x) Minimum requirement for connection density is $1,000,000$ devices per $\mathrm{km}^{2}$

(xi) Requirement for bandwidth is at least $100 \mathrm{MHz}$

(xii) Bandwidths up to $1 \mathrm{GHz}$ are required for higher frequencies (above $6 \mathrm{GHz}$ )

MR = Minimal Requirement

Source: ITU-R SG05 Contribution 40, "Minimum requirements related to technical performance for IMT-2020 radio interface(s)", Feb 2017.

(iv) Pragmatic deployment cost metrics, along with acceptable service price points across the gamut of applications and data rates, particularly in an IoT context.

Specifically, some of the design details are a latency below $5 \mathrm{msec}$. (as low as $1 \mathrm{msec}$.), support for device densities of up to 100 devices $/ \mathrm{m}^{2}$, reliable coverage area, integration of telecommunications services including mobile, fixed, optical and MEO/GEO satellite, and seamless support for the IoT ecosystem. For example, the technical objective $5 \mathrm{G}$ as envisioned of METIS (Mobile and Wireless Communications Enablers for the Twenty-twenty Information Society -- a European Community advocacy effort related to mobility) are as follows [47-54]:

(i) $1000 \mathrm{x}$ higher mobile data volume per area than current systems;

(ii) 10 to $100 \mathrm{x}$ higher number of devices than current systems (i.e., dense coverage);

(iii) 10 to $100 \mathrm{x}$ higher user data rate than current systems (e.g., 1-20 Gbps);

(iv) $10 \mathrm{x}$ longer battery life for low power IoT devices than current systems (up to a 10-year battery life for machine type communications); and,

(v) $5 \mathrm{x}$ reduced end-to-end latency than current systems.

Table 3 defines the $5 \mathrm{G}$ radio interface goals as defined in IMT2020. A number of these requirements are in fact being met (in various measure) by the systems now being deployed. The expectation is that to provide the full panoply of $5 \mathrm{G}$ services significant changes in both wireless technologies and core networks will be required.

As a point of observation, 3GPP/TR 22.891 has defined and/or described the following service groups: eMBB, Critical Communication, mMTC, Network Operations, and Enhancement of Vehicle-to-Everything (V2X). NGMN has defined and/or described the following service groups: Broadband access in dense area, Indoor ultra-high broadband access, Broadband access in a crowd, 50+ Mbps everywhere, Ultra low-cost broadband access for low ARPU areas,
Mobile broadband in vehicles, Airplanes connectivity, Massive low-cost Low long-range/low-power MTC, Broadband MTC, Ultra low latency, Resilience and traffic surge, Ultrahigh reliability and Ultra low latency, Ultra-high availability and reliability, and Broadcast-like services.

Figure 3 depicts some of the key $5 \mathrm{G}$ services that can be utilized for the IoT, in the medium term in Smart Cities; other services shown might also be used over time. Although some have associated Smart Cities with mMTC, we are of the opinion that the early applications will be more within the eMBB domain (some others also agree [55]). Also, one would expect eMBB to be deployed more broadly, driven by the commercial "appeal" of the video services it facilitates. Augmented and/or virtual reality (AR/VR) are emerging as keys application of $5 \mathrm{G}$ networks, also involving some IoT aspects. To meet the requirements of lower latency and massive data transmission in AR/VR applications, software-defined networking (SDN) with a multi-path cooperative route (MCR) scheme that minimizes delay may be ideally positioned for $5 \mathrm{G}$ small cell networks [56]. Note parenthetically that video requirements range from about $8 \mathrm{Mbps}$ for $\mathrm{HD}, 25 \mathrm{Mbps}$ for $\mathrm{UHD}$, $50 \mathrm{Mbps}$ for 360-degree UHD video, $200 \mathrm{Mbps}$ for 360degree HDR (high dynamic range) video, and up to 1 Gbps for $6 \mathrm{DoF} / \mathrm{MPEG}-\mathrm{I}$. The evolving MPEG-I Visual standard addresses visual technologies of immersive media; 360 video provides panoramic video texture projected onto a virtual shape surrounding the user's head, from which the user visualizes a portion for an immersive video experience; $6 \mathrm{DoF}$ (6 Degrees of Freedom) supports movements along three rotation axes and three translations and presumes that full freedom of movement through the scene is possible [57]. 5G/eMBB may eventually support some (but not necessarily all) of these video applications, but these applications are well beyond the IoT applications discussed in this paper. IP-based video surveillance in Smart Cities that may be supported by IoT can operate rather well at the 0.384-2.5 Mbps bandwidth range.

Figure 4 highlights some technical features of $5 \mathrm{G}$ services that can be utilized for the IoT in Smart Cities in terms of data rates, latency, reliability, device density and so on. 5G IoT overcomes the well-known limitation of unlicensed LPWAN 


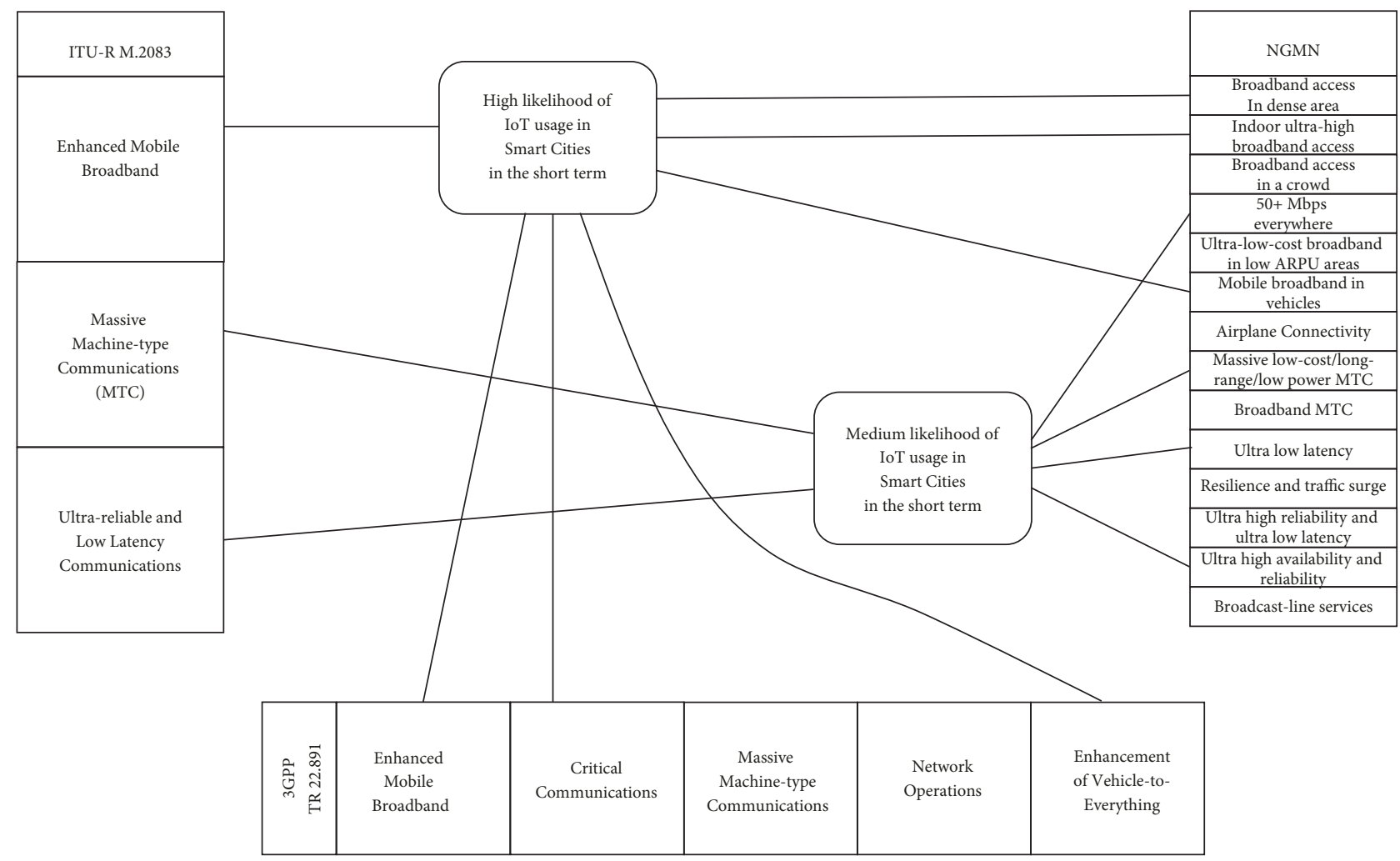

Figure 3: 5G services that can be utilized for the IoT in Smart Cities.

technologies that utilize crowded license-free frequency bands, especially in large cities; therefore, 5G IoT is ideal for Smart City for mission-critical and Quality of Service (QoS)aware applications (for example, traffic management, smart grid, utility control.)

2.2. $5 \mathrm{G}$ Evolution. $3 \mathrm{GPP}$ has specified new $5 \mathrm{G}$ radio access technology, 5G enhancements of $4 \mathrm{G}$ (fourth generation) networks, and new 5G core networks. Specifically, it has defined a new $5 \mathrm{G}$ Core network (5GC) and a new radio access technology called 5G "New Radio" (NR). The new 5GC architecture has several new capabilities built inherently into it as native capabilities: multi-Gbps support, ultra-low latency, Network Slicing, Control and User Plane Separation (CUPS), and virtualization. To deploy the 5GC new infrastructure will be needed. There is a firm goal to support for "forward compatibility". The 5G NR modulation technique and frame structure are designed to be compatible with LTE. The 5G NR duplex frequency configuration will allow 5G NR, NB-IoT and LTE-M subcarrier grids to be aligned. This will enable the $5 \mathrm{G}$ NR user equipment (UE) to coexist with NB-IoT and LTE-M signals. As might be expected, however, it is possible to integrate into $5 \mathrm{G}$ elements of different generations and different access technologies- two modes are allowed: the SA (standalone) configuration and the NSA (non-standalone) configuration (see Figure 5, also positioning IoT support).

(i) 5G Standalone (SA) Solution: in 5G SA an all new 5G packet core is introduced. SA scenarios utilize only one radio access technology (5G NR or the evolved
LTE radio cells); the core networks are operated independently.

(ii) 5G Non-Standalone Solution (NSA): in 5G NSA Operators can leverage their existing Evolved Packet Core (EPC)/LTE packet core to anchor the 5G NR using 3GPP Release 12 Dual Connectivity feature. This will enable operators to launch $5 \mathrm{G}$ more quickly and at a lower cost. This solution might suffice for some initial use cases. However, 5G NSA has a number of limitations, thus these Operators will eventually be expected to migrate to $5 \mathrm{G}$ Standalone solution. NSA scenario combines NR radio cells and LTE radio cells using dual-connectivity to provide radio access and the core network may be either EPC or $5 \mathrm{GC}$.

Multiple evolution/deployment paths may be employed by service providers (service providers of various services, including IoT services) to reach the final target configuration; this migration could well take a decade, and may also have different timetables in various parts of a country, e.g., top urban areas, top suburban areas, secondary urban areas, secondary suburban areas, exurbian areas, rural areas. Figure 6 depicts the well-known migration paths. The IoT implementer will need to be keenly aware of what 5G (5G IoT) services are available in a given area as an IoT implementation is contemplated. In Figure 6, Scenario 1 illustrates that the IoT Service provider will continue to use LTE and EPC to provide services (e.g., NB-IoT); here only legacy IoT devices can be supported. The provider only has a standalone radio 


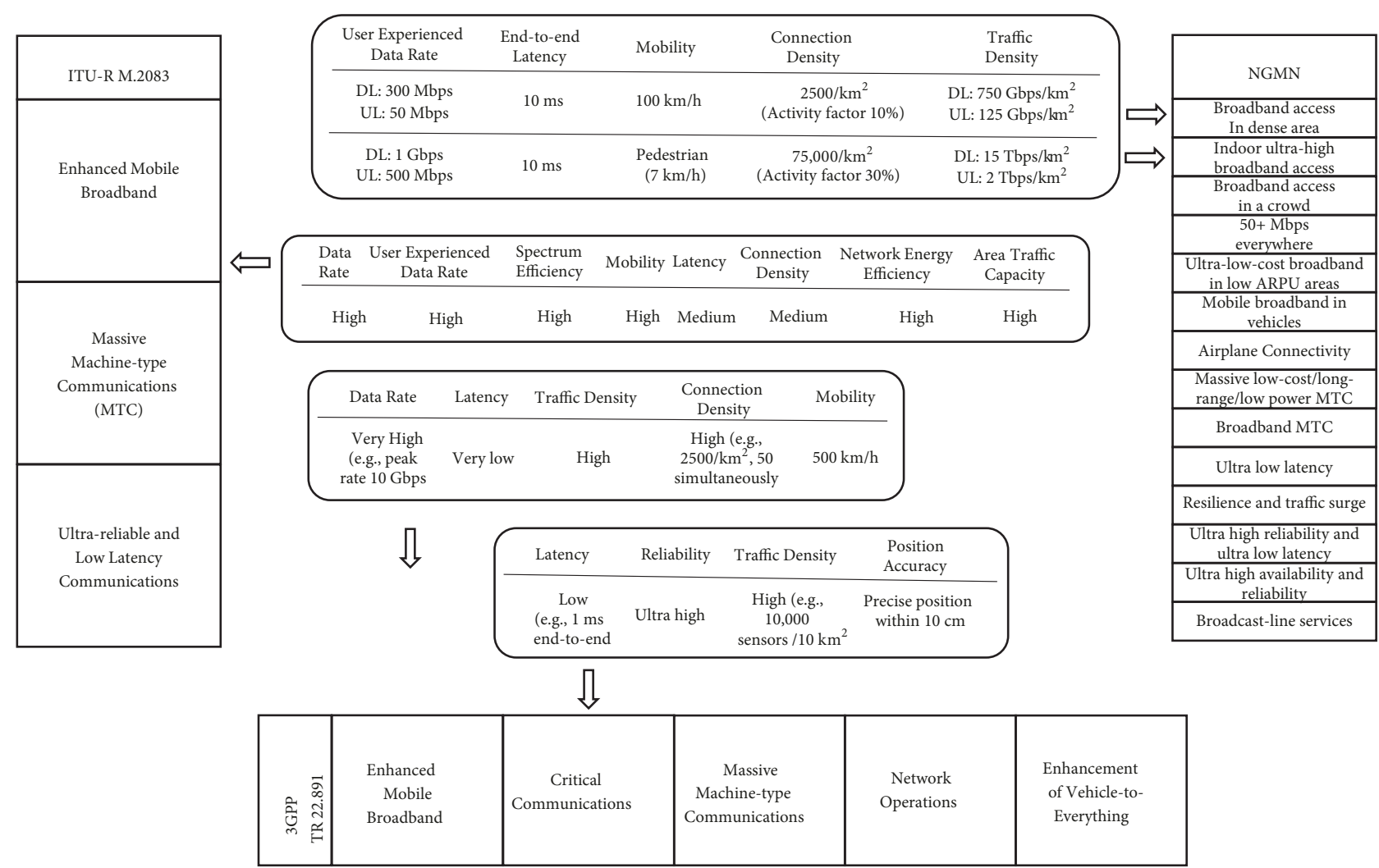

FIGURE 4: Some technical features of $5 \mathrm{G}$ services that can be utilized for the IoT in Smart Cities.

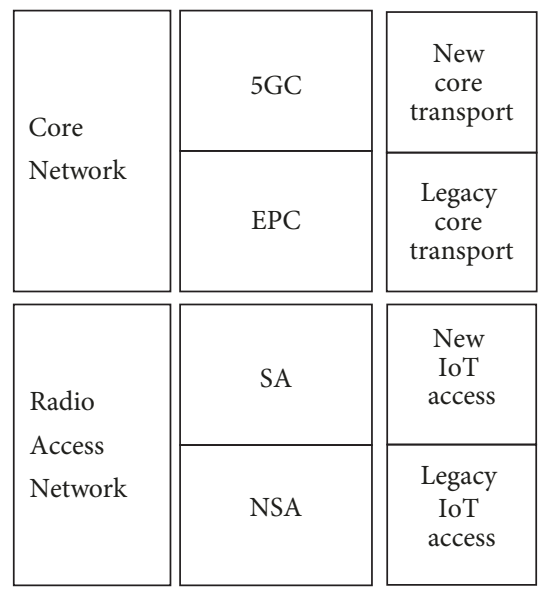

Core:

3GPP has defined a new $5 \mathrm{G}$ core network (5GC) and a new radio access Technology known as 5G "New Radio” (NR)

Access:

5G Standalone (SA) solution: In 5G SA an all new 5G packet core is introduced. SA scenarios utilize only one radio access technology (5G NR or the evolved LTE radio cells); the core networks are operated independently

5G Non-Standalone Solution (NSA): in 5G NSA, Operators can leverage their existing Evolved Packet Core (EPC)/LTE packet core to anchor the 5G NR using 3GPP Release 12 Dual Connectivity feature

FIgURE 5: 5G Transition Options and IoT support.

technology, in this case LTE only. Scenario 2 illustrates an IoT Service provider has migrated completely to NR (again only providing a standalone radio technology), but will retain the existing core network, the EPC. (Only) new 5G IoT devices can be used. In scenarios 5 and 6 the service providers will support both the legacy LTE and the new NR (clearly in this non-standalone arrangement, both radio technologies are deployed.) Some of these providers retain the legacy core and some will deploy the new 5GC core. Both legacy and 5G IoT devices can be supported.
3GPP approved the 5G NSA standard at the end of 2017 and the 5G SA standard in early 2018 in the context of its Release 15. Release 15 also included the support eMBB, URLLC, and mMTC in a single network to facilitate the deployment of IoT services; Release 15 also supports $28 \mathrm{GHz}$ millimeter-wave (mmWave) spectrum and multi-antenna technologies for access.

2.3. 5G Frequency Bands. Focusing on the radio technology, there are number of spectrum bands that can be used in 

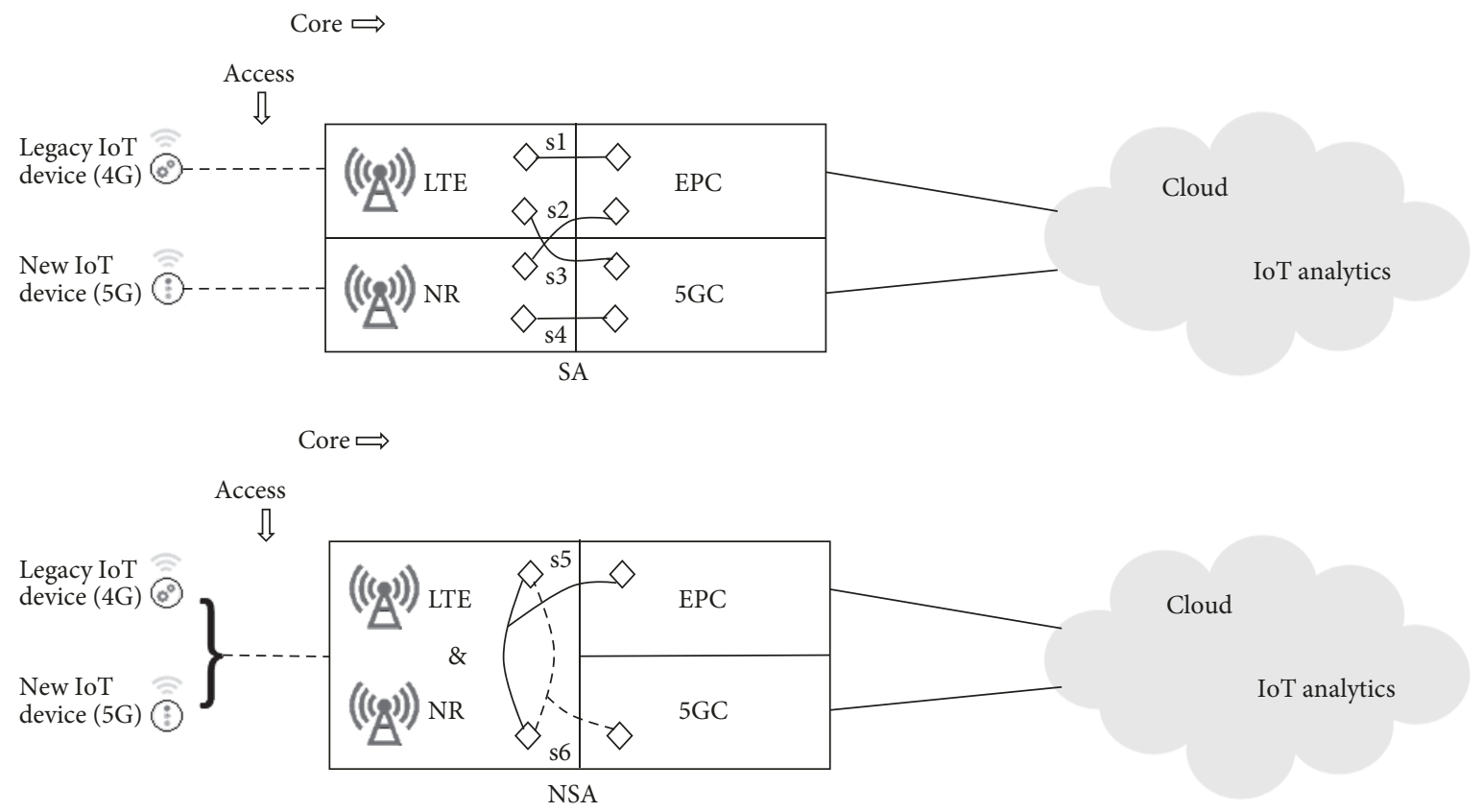

FIgURE 6: Detailed 5G Transition Options and IoT support.

5G; these bands can be grouped into three macro categories: sub-1 GHz, 1-6 GHz and above $6 \mathrm{GHz}$. The more advanced features, especially higher data rates require the use of the millimeter wave spectrum. New mobile generations are typically assigned new frequency bands and wider spectral bandwidth per frequency channel (1G up to $30 \mathrm{kHz}, 2 \mathrm{G}$ up to $200 \mathrm{kHz}, 3 \mathrm{G}$ up to $5 \mathrm{MHz}$, and $4 \mathrm{G}$ up to $20 \mathrm{MHz}$ ). Up to now cellular networks have used frequencies below $6 \mathrm{GHz}$. Generally, without advanced MIMO (Multiple In Multiple Out) antenna technologies one can obtain about 10 bits-per-Hertz-of-channel bandwidth. But the integration of new radio concepts such as Massive MIMO, Ultra Dense Networks, Device-to-Device, and mMTC will allow 5G to support the expected increase in the data volume in mobile environments and facilitate new IoT applications. Implementable standards for $5 \mathrm{G}$ are being incorporated in 3GPP Release 15 onwards. As noted, 3GPP Rel 15 defines New 5G Radio and Packet Core evolution to facilitate interoperable deployment of the technology.

The millimeter wave spectrum, also known as Extremely High Frequency (EHF), or more colloquially mmWave, is the band of electromagnetic spectrum running between 30 $\mathrm{GHz}$ and $300 \mathrm{GHz}$. Bands within this spectrum are being considered by the ITU and the Federal Communications Commission in the U.S. as a mechanism to facilitate $5 \mathrm{G}$ by supporting higher bandwidth. The use of a $3.5 \mathrm{GHz}$ frequency to support $5 \mathrm{G}$ networks is also gaining some popularity, but he higher speeds networks will use other frequency bands, including millimeter-wave frequencies (these bands ranging from $28 \mathrm{GHz}$ to $73 \mathrm{GHz}$, specifically the 28, 37, 39, 60 and $72-73 \mathrm{GHz}$ bands). In the U.S., recently the FCC approved spectrum for $5 \mathrm{G}$, including millimeter-wave frequencies in the $28 \mathrm{GHz}, 37 \mathrm{GHz}$ and $39 \mathrm{GHz}$ bands, although these targeted cellular frequencies may nominally overlap with other pre-existing users of the spectrum, for example point-to-point microwave paths, Direct Broadcast satellite TV, and high throughput satellite (HTS) systems (Kaband transmissions).

Initially $5 \mathrm{G}$ will, in many cases, use the $28 \mathrm{GHz}$ band, but higher bands will very likely be utilized later on; initial implementations, will support a maximum speed of 1 Gbps. Lower frequencies (at the so-called C band) are less subject to weather impairments, can travel longer distances, and penetrate building walls more easily. Waves at higher frequencies ( $\mathrm{Ku}, \mathrm{Ka}$ and $\mathrm{E} / \mathrm{V}$ bands) do not naturally travel as far or penetrate walls or objects as easily. However, a lot more channel bandwidth is available in millimeter-wave bands. Furthermore, developers see the need for "an innovative utilization of spectrum"; "small cell" approaches are required to address the scarcity of the spectrum, but at the same time covering the geography. V band spectrum covers $57-71 \mathrm{GHz}$, which in many countries is an "unlicensed" band, and E band spectrum covers $71-76 \mathrm{GHz}, 81-86 \mathrm{GHz}$ and $92-95 \mathrm{GHz}$.

In the U.S., in 2018 the FCC also opened up, as an "interim" step for 5G, a "mid-band" radio spectrum at $3.5 \mathrm{GHz}$ which was previously reserved for naval radar use. The $3.5 \mathrm{GHz}$ band provides a combination of signal propagation distance, acceptable building penetration, and increased bandwidth. The FCC created 15 channels within the 3.550-3.700 GHz band, auctioning seven channels to "priority access licenses" and making eight channels available for general access -- the U.S. Navy still getting priority across the band when and as needed. With this approval, 5G devices can be built to support the same $3.5 \mathrm{GHz}$ ranges across North America, Europe, and Asia [58].

In addition to new bands, $5 \mathrm{G}$ technology is expected to use beam-forming and beam-tracking, where a cell's antenna can focus its signal to reach a specific mobile device and 


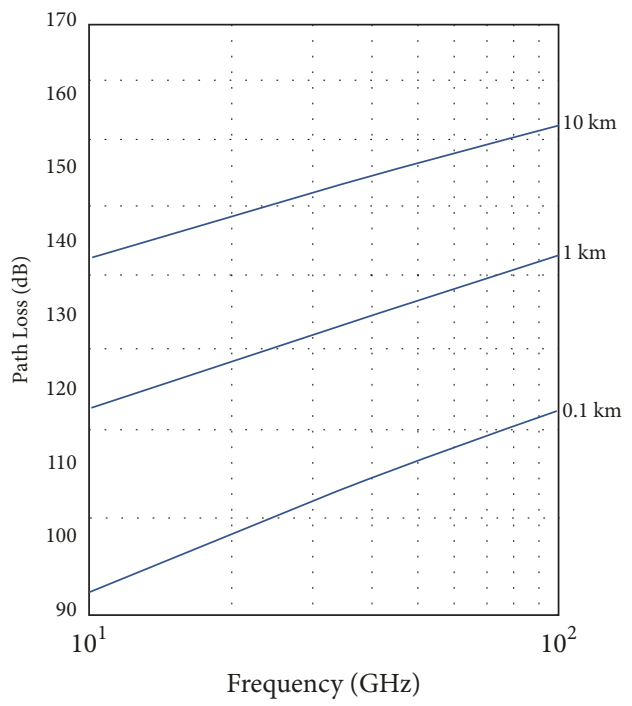

Figure 7: Path loss as a function of distance and frequency.

then track that device as it moves. Beamforming utilizes a large number (hundreds) of antennas at a base station to achieve highly directional antenna beams that can be "steered" in a desired direction to optimize transmission and throughput performance. Massive MIMO is a system where a transmission node (base station) is equipped with a large number (hundreds) of antennas that simultaneously serve multiple users; with this technology multiple messages for several terminals can be transmitted on the same timefrequency resource.

\subsection{G Transmission Characteristics at Higher Frequencies.} Due to RF propagation phenomena that are more pronounced at the higher frequencies, such as multipath propagation due to outdoor and indoor obstacles, free space path loss, atmospheric attenuation due to rain, fog, and air composition (e.g., oxygen), small cells will almost invariably be needed in 5G environments, especially in dense urban environments. Additionally, Line of Sight (LOS) will typically be required. ITU-R P series of recommendations has useful information on radio wave propagation including ITU-R P.838-3, 2005; ITU-R P.840-3, 2013; ITU-R P.676-10, 2013; and ITU-R P.525-2, 1994. Figures 7, 8, 9, and 10 highlight the issues at the higher frequencies, including the millimeterwave frequencies. Figure 7 depicts the path loss as a function of distance and frequency. Figure 8 shows the attenuation as a function of precipitation and frequency. Figure 9 illustrates the attenuation as a function of fog density and frequency. Figure 10 depicts the attenuation as a function of atmospheric gases and frequency (notice high attenuation around 60 $\mathrm{GHz}$ ).

In addition to the broad service requirements briefly highlighted in Table 3 (for example latency, user density distribution, etc.), there are specific IoT nodal considerations that have to be taken into account as one develops the next generation network. For example, IoT nodes typically are low-complexity devices and have limited on-board power. $5 \mathrm{G}$ systems have to take these restrictions and considerations

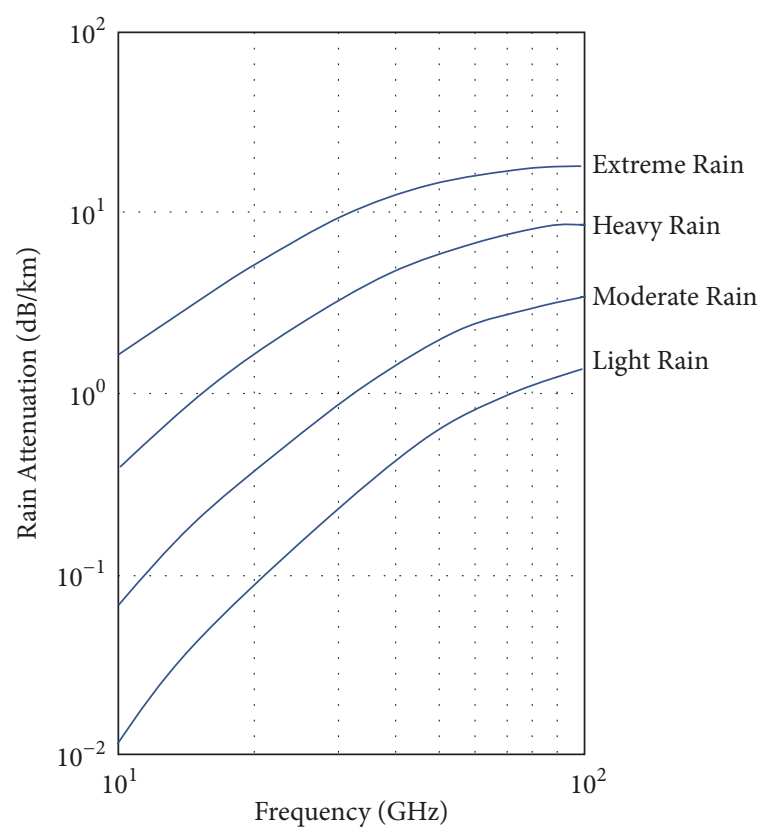

FIGURE 8: Attenuation a function of precipitation and frequency.

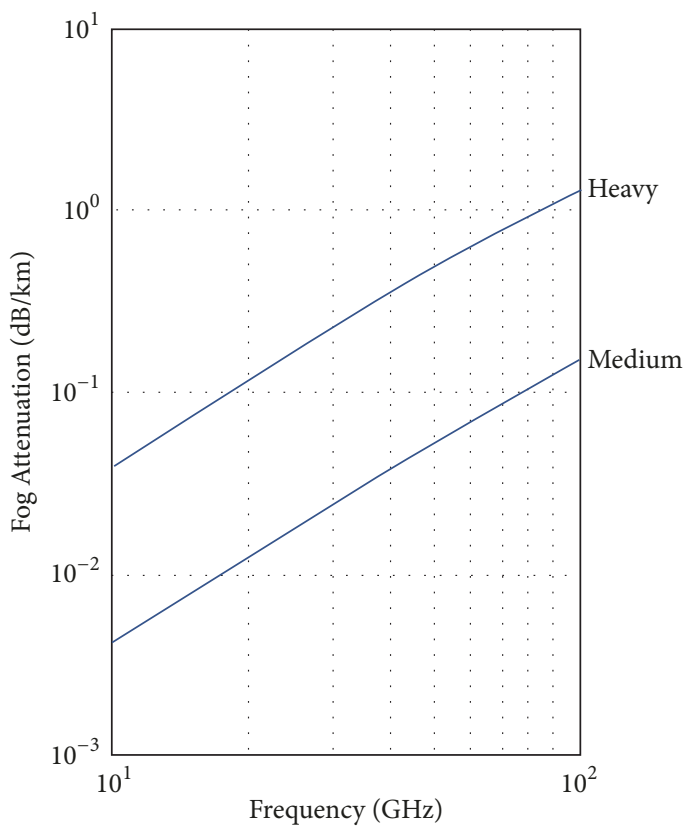

FIgURE 9: Attenuation a function of fog density and frequency.

into account. Table 4 provides a summary of some of these considerations and the $5 \mathrm{G}$ support.

\section{Small Cell and Building Penetration Issues}

As expected, communications at mmWave frequencies have attracted a lot of interest, due to the large available spectrum bandwidth that can potentially result in multiple gigabit per second transmissions per user. This follows a similar trend 


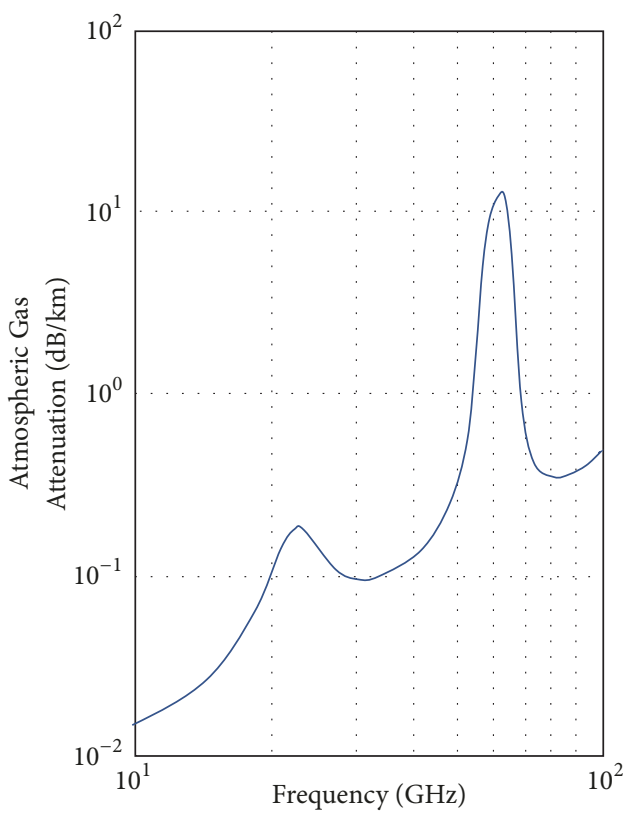

FIGURE 10: Attenuation a function of atmospheric gases and frequency. (notice high attenuation around $60 \mathrm{GHz}$ ).

in satellite communications with the introduction of Ka services, especially HTSs. High bandwidth will typically require a wide spectrum. Millimeter wave frequencies (signals with wavelength ranging from 1 millimeter to 10 millimeters) support a wide usable spectrum. The millimeter wave spectrum includes licensed, lightly licensed and unlicensed portions. Bandwidth demand and goals are the main driver for the need to use the millimeter wave spectrum, particularly for eMBB-based applications, allowing users to receive $100 \mathrm{Mbps}$ as a bare minimum and 20 Gbps as a theoretical maximum. The use of millimeter wave frequencies, however, will imply the use of a much smaller tessellation of cells and supportive towers or rooftop transmitters due, as noted, to transmission characteristics, such as high attenuation and directionality. This is an important design consideration for $5 \mathrm{G}$, especially in dense city/urban environments. The aggregation of these towers will by itself require a significant backbone network, whether a mesh based on some point-to-point microwave links, an fiber network or a set of "wireless fiber" links. Millimeter wave system utilize smaller antennas compared to systems operating at lower frequencies: the higher frequencies, in conjunction with MIMO techniques, can achieve sensible antenna size and cost. The millimeter wave technology can be utilized both for indoors and outdoors highcapacity fixed or mobile communication applications. The term "densification" is also used to describe the massive deployment of small cells in the near future.

MmWave products used for backhauling typically operate at $60 \mathrm{GHz}$ (V Band) and 70/80 GHz (E Band) and offer solutions in both Point to Point and Point to Multipoint (PtMP) configurations providing end to end multi-gigabit wireless networks, for example, 1 Gbps up to 10 Gbps symmetric performance. Very small directional antennas, typically less than a half-square foot in area, are used to transmit and/or receive signals which are highly focused beams. stationary radio systems are often installed on rooftops or towers. MmWave products are now appearing on the market targeting high capacity Smart City applications, 5G Fixed, Gigabit Wireless Access solutions, and Business Broadband. Urban canyons, however, may limit the utility of this technology to very short LOS paths. Mobile applications of mmWave technology are more challenging. On the other hand, one advantage of this technology is that short transmission paths (high propagation losses) and high directionality allow for spectrum reuse by limiting the amount of interference between transmitters and/or adjacent cells. Near LOS (NLOS) applications may be possible in some cases (especially for short distances).

Currently, $\mathrm{mm}$ wave frequencies are being utilized for high-bandwidth indoor applications, for example streaming ("miracasting") of HD or UHD video and VR support (e.g., using 802.11ad Wi-Fi). Traditionally, these frequencies have not been used for outdoor broadband applications due to high propagation loss, multipath interference, and atmospheric absorption (gases, rain, fog and humidity) cited above; in addition, the practical transmission range is a few kilometers in open space [68]. Recently the FCC proposed new rules for wireless broadband in wireless frequencies above $24 \mathrm{GHz}$ stating that it is "taking steps to unlock the mobile broadband and unlicensed potential of spectrum at the frontier above $24 \mathrm{GHz}$ " [69]. The ITU and the 3GPP have defined two-phases of research; the first phase (expected to complete by press time) is to assess frequencies less than $40 \mathrm{GHz}$ to address short-term commercial requirements; the second phase entails assessing the IMT 2020 requirements by studying frequencies up to $100 \mathrm{GHz}$. The following mmWave bands being considered, among other bands [70]:

(i) $7 \mathrm{GHz}$ of spectrum in total in the band $57 \mathrm{GHz}$ to 64 $\mathrm{GHz}$ unlicensed.

(ii) $3.4 \mathrm{GHz}$ of spectrum in total in the $28 \mathrm{GHz} / 38 \mathrm{GHz}$ licensed but underutilized region.

(iii) $12.9 \mathrm{GHz}$ of spectrum in total $71 \mathrm{GHz} / 81 \mathrm{GHz} / 92 \mathrm{GHz}$ light-licensed band

Following the most recent World Radiocommunications Conference, the ITU also identified a list of proposed globally-usable frequencies between $24 \mathrm{GHz}$ and $86 \mathrm{GHz}$, as follows: $24.25-27.5 \mathrm{GHz}, 31.8-33.4 \mathrm{GHz}, 37-40.5 \mathrm{GHz}$, 40.5-42.5 GHz, 45.5-50.2 GHz, 50.4-52.6 GHz, 66-76 GHz, and $81-86 \mathrm{GHz}$.

3.1. Cell Types. MmWave transmission will drive the requirement for small cells $[71,72]$. "Small cells" refer to relatively low-powered radio communications equipment (base stations) and ancillary antennas and/or towers that provide mobile, internet, and IoT services within localized areas. Small cells typically have a range up to one-to-two kilometers, but can also be smaller -- on the other hand, a typical mobile macrocell (such as urban macro-cellular [UMa] or rural macrocell $[\mathrm{RMa}])$ has a range of several kilometers up to 10to-20 of kilometers.) The terms femtocells, picocells, microcells, urban microcell (UMi), and metrocells are effectively synonymous with the "small cells" concept. Small(er) cells 
TABLE 4: Example of IoT nodal considerations for $5 \mathrm{G}$ systems.

\begin{tabular}{|c|c|}
\hline IoT device issue & 5G Support \\
\hline Low complexity devices & $\begin{array}{l}\text { Broad standardization leads to simplification e.g., SOC (System on a Chip) } \\
\text { and/or ASIC (Application Specific IC) development }\end{array}$ \\
\hline Limited on-board power & Technology allows a battery life $\sim 10$ years \\
\hline Device mobility & Good mobility support in a cellular/5G system \\
\hline Open environment & Broad standardization leads to broad acceptance of the technology \\
\hline $\begin{array}{l}\text { Devices universe by type and by } \\
\text { cardinality }\end{array}$ & $\begin{array}{l}\text { Standardized air interfaces can reduce certain aspects of the end-node, just } \\
\text { like Ethernet simplified connectivity to a network, regardless of the } \\
\text { functionality of the processor per se }\end{array}$ \\
\hline $\begin{array}{l}\text { Always connected/always on mode of } \\
\text { operation }\end{array}$ & Cost-effective connectivity services allow the always on mode of operation \\
\hline IoT security (IoTSec) concerns $[59,60]$ & $\begin{array}{l}\text { Security capabilities are being added. The use of 256-bit symmetric } \\
\text { cryptography mechanisms is expected to be fully incorporated. The } \\
\text { encryption algorithms are based on SNOW 3G, AES-CTR, and ZUC; and } \\
\text { integrity algorithms are based on SNOW 3G, AES-CMAC, and ZUC. The } \\
\text { main key derivation function is based on HMAC-SHA-256. Identity } \\
\text { management (e.g., via the 5G authentication and key agreement [5G AKA] } \\
\text { protocol and/or the Extensible Authentication Protocol [EAP]), Privacy } \\
\text { (conforming to the General Data Protection Regulation [GDPR]), and } \\
\text { Security assurance (e.g., using Network Equipment Security Assurance } \\
\text { Scheme [NESAS]) are supported. Some of these mechanisms are described } \\
\text { [61-65]. As another example, the ETSI Technical Committee on } \\
\text { Cybersecurity issued in } 2018 \text { two encryption specifications for access } \\
\text { control in highly distributed systems such as G and IoT. Attribute-Based } \\
\text { Encryption (ABE) that describes how to secure personal data. }\end{array}$ \\
\hline $\begin{array}{l}\text { Lack of agreed-upon end-to-end } \\
\text { standards }\end{array}$ & $\begin{array}{l}\text { Broad standardization possible with } 5 \mathrm{G} \text { if the technology is broadly } \\
\text { deployed and is cost-effective }\end{array}$ \\
\hline $\begin{array}{l}\text { Lack of agreed-upon end-to-end } \\
\text { architecture }\end{array}$ & $\begin{array}{l}\text { Standardization at the lower layers (Data Link Control and Physical) can } \\
\text { drive the development of a more inclusive multi-layer multi-application } \\
\text { architecture }\end{array}$ \\
\hline
\end{tabular}

have been used for years to increase area spectral efficiency -- the reduced number of users per cell provides more usable spectrum to each user. However, the smaller cells in 5G are also dictated by the propagation characteristics. In the $5 G$ context UMi typically have radii of 5-120 meters for LOS and 20 to 270 meters in NLOS; UMa typically have radii of 60-1000 meters for LOS and 50-1500 meters for NLOS [73]. Given their size, 5G/mmWave UMi cells will be able to support high bandwidth enabling eMBB services over small areas of high traffic demand. At the mmWave operation, userdevice proximity with the antenna will enable higher signal quality, lower latency, and by definition, high data rates and throughput. Also, to be noted, mmWave frequencies make the size of multi-element antenna arrays practical, enabling large Multi-user MIMO (MU-MIMO) solutions.

Signal penetration indoors may represent a challenge, just as is the case even at present with $3 \mathrm{G} / 4 \mathrm{G}$ LTE, even for traditional voice and internet access and data services. This has driven the need for DAS systems, especially in denselyconstructed downtown districts. Free space attenuation at the higher frequency, power budgets, directionality requirements, and weather, all impact 5G and 5G IoT. Outdoor small cells and building-resident Distributed Antenna Systems (DAS) systems utilize high-speed fiber optic lines or "wireless fiber" to interconnect the sites to the backbone and the Internet cloud.
Figure 11 depicts a 5G IoT ecosystem where mmWave technology is used. Figure 12 shows typical (4G LTE) urban microcell towers. Figure 13 depicts a Smart City supported via (5G) urban microcells.

3.2. Assessment of Transmission Issues. Reference [74] provides a fairly comprehensive assessment of the transmission channel issues as they apply to $5 \mathrm{G}$. The importance of this topic is accentuated by the large number of agencies actively researching this topic, including [55, 73-87]:

(i) METIS

(ii) $3 \mathrm{GPPP}$

(iii) MiWEBA (Millimetre-Wave Evolution for Backhaul and Access)

(iv) ITU-R M

(v) COST2100

(vi) IEEE 802.11

(vii) NYU WIRELESS: interdisciplinary academic research center

(viii) IEEE $802.11 \mathrm{ad} / \mathrm{ay}$

(ix) QuaDRiGa (Fraunhofer HHI)

(x) 5th Generation Channel Model (5GCM) 


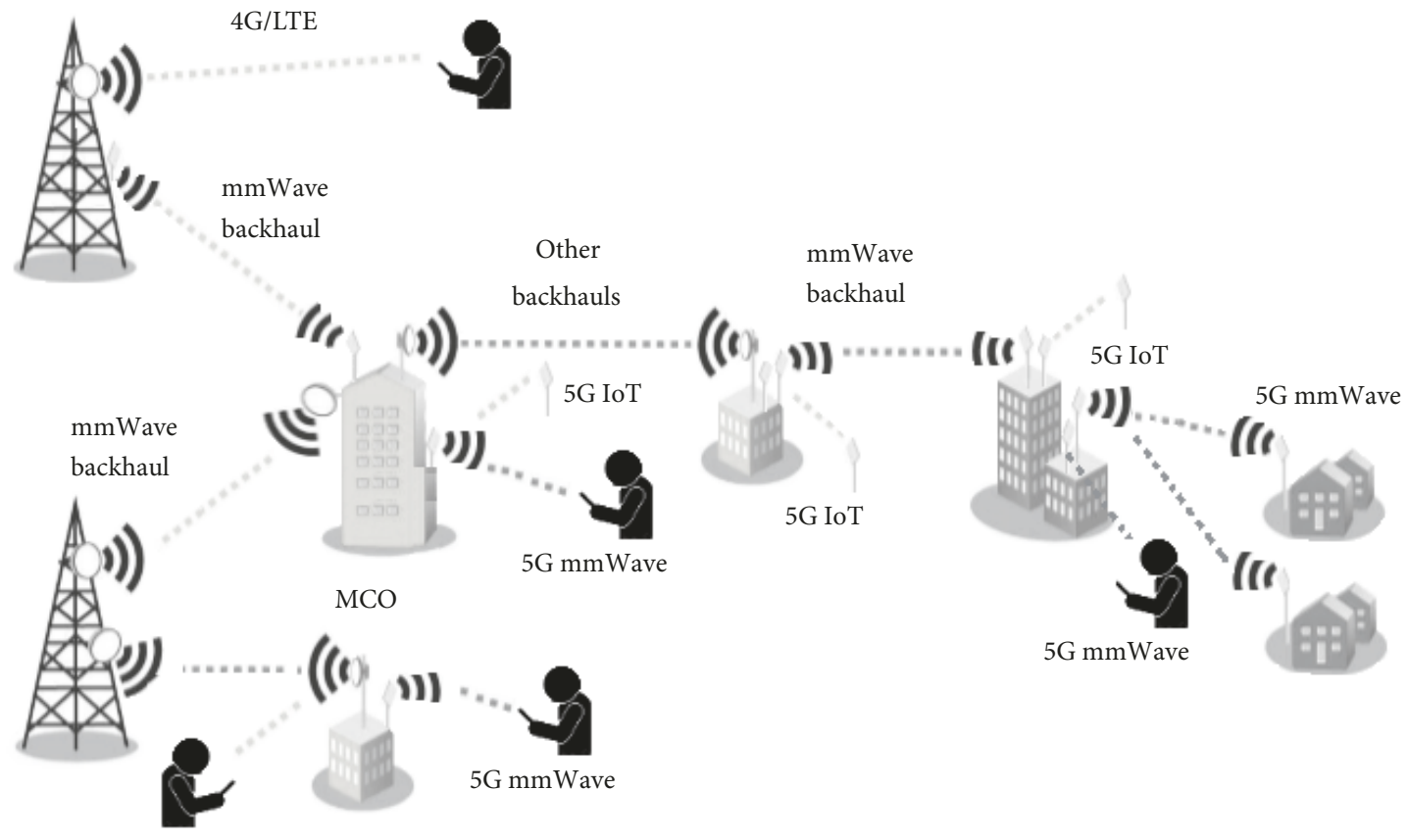

$5 \mathrm{G}$ 'midband'

FIgURE 11: The 5G IoT ecosystems.

Microcell towers, usable in 5G and 5G IoT
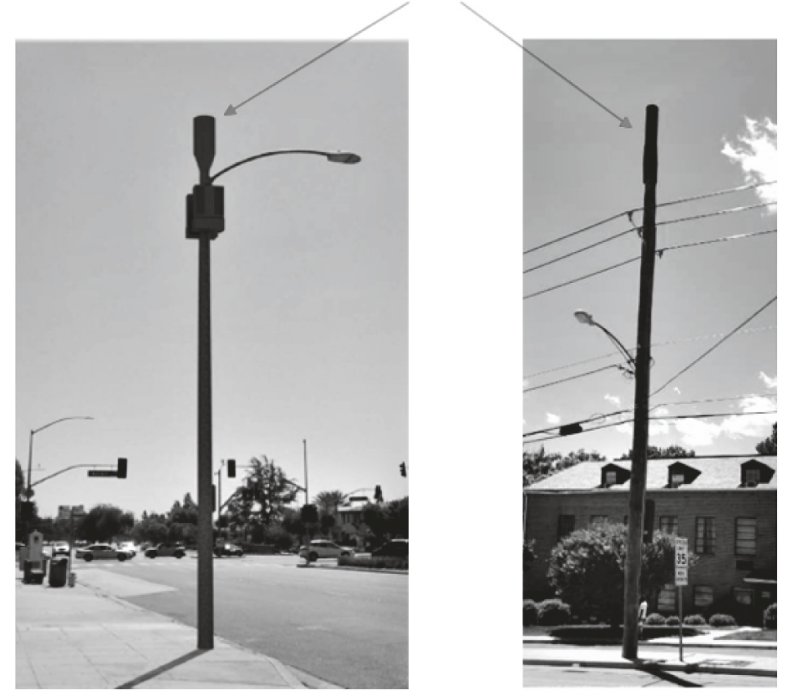

FIGURE 12: Microcell towers (these for $4 G$, but a lot more for $5 G$ ). (noncopyrighted material from FCC-related filings [91]).

(xi) 5G mmWave Channel Model Alliance (NIST initiated, North America based)

(xii) mmMAGIC (Millimetre-Wave Based Mobile Radio Access Network for Fifth Generation Integrated Communications) (Europe based)

(xiii) IMT-2020 5G promotion association (China based)

(also including firms and academic centers such as, but not limited to AT\&T, Nokia, Ericsson, Huawei, Intel/Fraunhofer

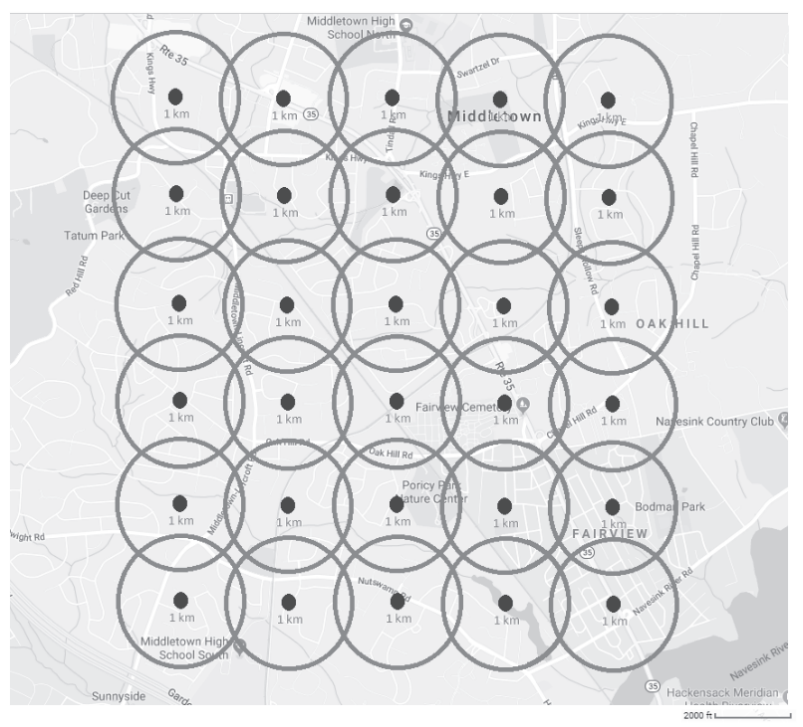

Figure 13: Microcells for 5G/5G IoT.

HHI, NTT DOCOMO, Qualcomm, CATT, ETRI, ITRI/CCU, ZTE, Aalto University, and CMCC.)

Diffraction loss (DL) and frequency drop (FD) are just two of the path quality issues to be addressed. Although greater gain antennas will likely be used to overcome path loss, diffuse scattering from various surfaces may introduce large signal variations over travel distances of just a few centimeters, with fade depths of up to $20 \mathrm{~dB}$ as a receiver moved by a few centimeters. These large variations of the channel must be taken into consideration for reliable design 


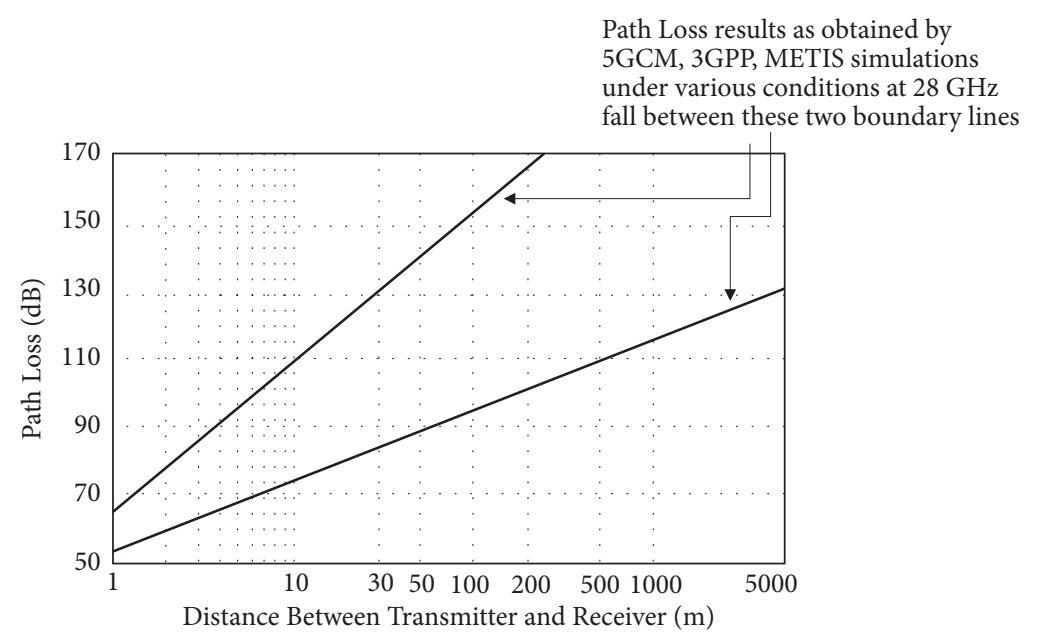

FIGURE 14: Path Loss simulations for $5 \mathrm{G}$ by various entities.

of channel performance, including beam-forming/tracking algorithms, link adaptation schemes, and state feedback algorithms. Furthermore, multipath interference from coincident signals can give rise to critical small-scale variations in the channel frequency response. In particular, wave reflection from rough surfaces will cause high depolarization. For LOS environment Rician fading of multipath components, exponential decaying trends and quick decorrelation in the range of 2.5 wavelengths have been demonstrated. Furthermore, received power of wideband mmWave signals has a stationary value for slight receiver movements but average power can change by $25 \mathrm{~dB}$ as the mobile transitions around a building corner from NLOS to LOS in an UMi setting. Additionally, human body blockage causes more than $40 \mathrm{~dB}$ of fading at the mmWave frequencies. Figure 14 depicts the path loss according to various simulations for $5 \mathrm{G}$ by various stakeholder entities.

The main parameter of the radio propagation model is the Path Loss Exponent (PLE), which is an attenuation exponent for the received signal. PLE has a significant impact on the quality of the transmission links. In the far field region of the transmitter, if $\mathrm{PL}\left(\mathrm{d}_{0}\right)$ is the path loss measured in $\mathrm{dB}$ at a distance $d_{0}$ from the transmitter, then the loss in signal power expected when moving from distance $d_{0}$ to $d\left(d>d_{0}\right)$ is [8890] is

$$
\begin{aligned}
P L_{d_{0} \rightarrow d}(d B)=P L\left(d_{0}\right)+10 n \log _{10}\left(\frac{d}{d_{0}}\right) & +\chi \\
& d_{f} \leq d_{0} \leq d
\end{aligned}
$$

where

$$
\operatorname{PL}\left(\mathrm{d}_{0}\right)=\text { Path Loss in } \mathrm{dB} \text { at a distance } \mathrm{d}_{0}
$$

$\mathrm{n}=\mathrm{PLE}$

$\chi=$ A zero-mean Gaussian distributed random variable with standard deviation $\sigma$. (This is utilized only when there is a shadowing effect; if there is no shadowing effect, then this random variable is taken to be zero.)
See Figure 15. Usually PLE is considered to be known upfront but in most instances PLE needs to be assessed for the case at hand. It is advisable to estimate the PLE as accurately as possible for the given environment. PLE estimation is achieved by comparing the observed values over a sample of measurements to the theoretical values. Obstacles absorb signals, thus treating the PLE as a constant is not an accurate representation of the real environments, both indoors and outdoors (for example treating PLE as a constant which may cause serious positioning errors in complicated indoor environments [88]). Usually to model real environments the shadowing effects cannot be overlooked, by taking the PLE as a constant (a straight-line slope). To capture a shadowing effect a zero-mean Gaussian random variable with standard deviation $\sigma$ is added to the equation. Here the PLE (slope) and the standard deviation of the random variable should be known precisely for a better modeling.

Table 5 provides theoretical performance equations developed by 3GPP and ETSI for outdoor channel performance [81]. As pragmatic working parameters, one has the following:

(i) PLE values are in the 1.9 and 2.2 range for LOS and at the $28 \mathrm{GHz}$ and $60 \mathrm{GHz}$ bands; PLE is approximately 4.5 and 4.2 range for NLOS in the $28 \mathrm{GHz}$ and $60 \mathrm{GHz}$ bands.

(ii) Rain attenuation of $2-20 \mathrm{~dB} / \mathrm{km}$ can be anticipated for rain events ranging from light rain $(12.5 \mathrm{~mm} / \mathrm{hr})$ to downpours $(50 \mathrm{~mm} / \mathrm{hr}$ ) at $60 \mathrm{GHz}$ (higher for tropical events). For 200-meter cells, the attenuation will be around $0.2 \mathrm{db}$ for $5 \mathrm{~mm} / \mathrm{hr}$ rain at $28 \mathrm{GHz}$ and $0.9 \mathrm{~dB}$ for $25 \mathrm{~mm} / \mathrm{hr}$ rain at $28 \mathrm{GHz}$. The attenuation will be around $0.5 \mathrm{db}$ for $5 \mathrm{~mm} / \mathrm{hr}$ rain at $60 \mathrm{GHz}$ and $2 \mathrm{~dB}$ for $25 \mathrm{~mm} / \mathrm{hr}$ rain at $60 \mathrm{GHz}$.

(iii) Atmospheric absorption of $1-10 \mathrm{~dB} / \mathrm{km}$ occurs at the mmWave frequencies. For 200-meter cells the absorption will be $0.04 \mathrm{~dB}$ at $28 \mathrm{GHz}$ and $3.2 \mathrm{~dB}$ at $60 \mathrm{GHz}$. 
TAble 5: Path Loss Equations for mmWave 5G/5G IoT.

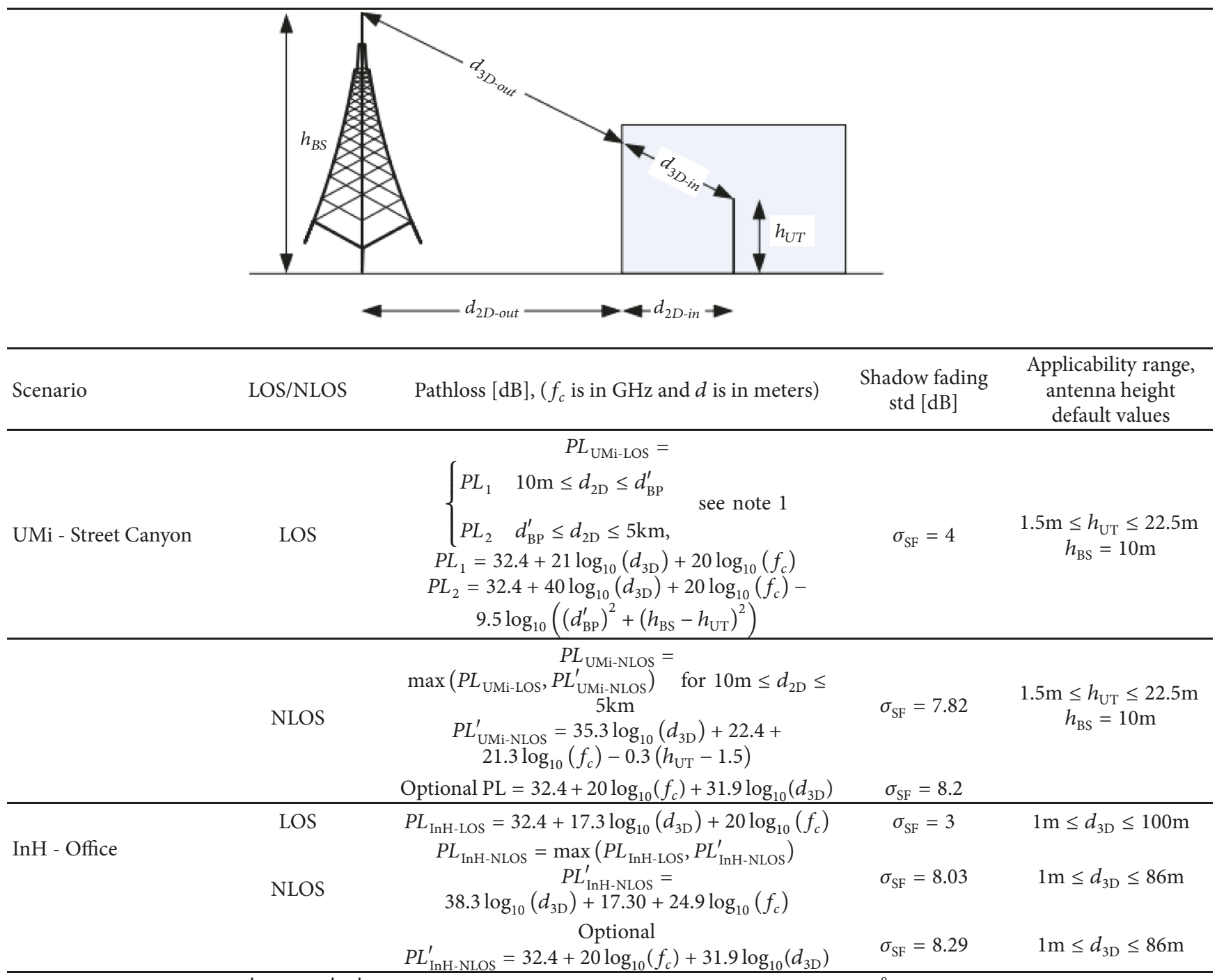

Note 1: Breakpoint distance $d_{\mathrm{BP}}^{\prime}=4 h_{\mathrm{BS}}^{\prime} h_{\mathrm{UT}}^{\prime} f_{c} / c$, where $f_{c}$ is the centre frequency in $\mathrm{Hz}, c=3.0 \times 10^{8} \mathrm{~m} / \mathrm{s}$ is the propagation velocity in free space, and $h_{\mathrm{BS}}^{\prime}$ and $h_{\mathrm{UT}}^{\prime}$ are the effective antenna heights at the BS and the UT, respectively. The effective antenna heights $h_{\mathrm{BS}}^{\prime}$ and $h_{\mathrm{UT}}^{\prime}$ are computed as follows: $h_{\mathrm{BS}}^{\prime}=h_{\mathrm{BS}}-h_{\mathrm{E}}, h_{\mathrm{UT}}^{\prime}=h_{\mathrm{UT}}-h_{\mathrm{E}}$, where $h_{\mathrm{BS}}$ and $h_{\mathrm{UT}}$ are the actual antenna heights, and $\mathrm{h}_{\mathrm{E}}$ is the effective environment height. For UMi $h_{\mathrm{E}}=1.0 \mathrm{~m}$. For Uma $h_{\mathrm{E}}=1 \mathrm{~m}$ with a probability equal to $1 /\left(1+\mathrm{C}\left(d_{2 \mathrm{D}}, h_{\mathrm{UT}}\right)\right)$ and chosen from a discrete uniform distribution uniform $\left(12,15, \ldots,\left(h_{\mathrm{UT}}-1.5\right)\right)$ otherwise. With $\mathrm{C}\left(d_{2 \mathrm{D}}, h_{\mathrm{UT}}\right)$ given by $C\left(d_{2 \mathrm{D}}, h_{\mathrm{UT}}\right)=\left\{0, h_{\mathrm{UT}}<13 \mathrm{~m} ;\left(\left(h_{\mathrm{UT}}-13\right) / 10\right)^{1.5} g\left(d_{2 \mathrm{D}}\right), 13 \mathrm{~m} \leq h_{\mathrm{UT}} \leq 23 \mathrm{~m}\right\}$ where $g\left(d_{2 \mathrm{D}}\right)=\left\{0, d_{2 \mathrm{D}} \leq 18 \mathrm{~m} ;(5 / 4)\left(d_{2 \mathrm{D}} / 100\right)^{3} \exp \left(-d_{2 \mathrm{D}} / 150\right), 18 \mathrm{~m}<d_{2 \mathrm{D}}\right\}$

3GPP TR 38.901 version 14.0.0 Release 14

ETSI TR 138901 V14.0.0 (2017-05).

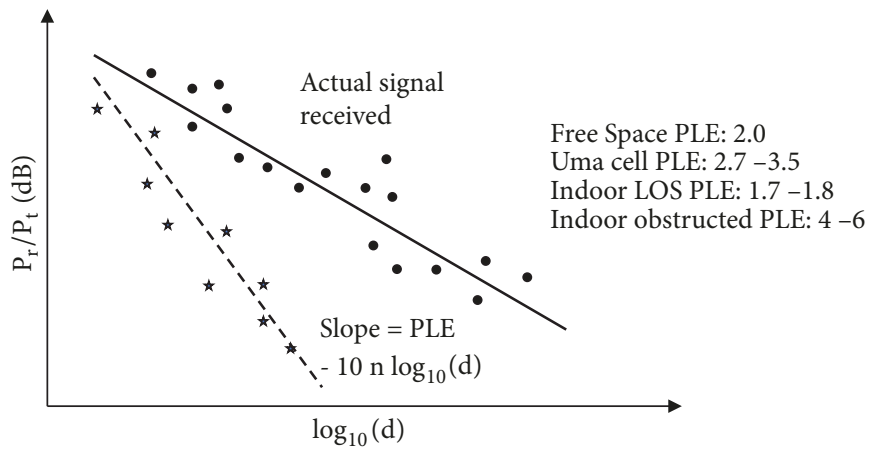

FIGURE 15: PLE. 
Penetration into buildings is an issue for mmWave communication, this being a lesser concern for contemporary sub 1 $\mathrm{GHz}$ systems and even systems operating up to $6 \mathrm{GHz}$. O2I (Outdoor-to- Indoor) losses have to be taken into account. Actual measurements (e.g., at $38 \mathrm{GHz}$ ) demonstrated a penetration loss of $40 \mathrm{~dB}$ for brick pillars, $37 \mathrm{~dB}$ for a glass door, and $25 \mathrm{~dB}$ for a tinted glass window (indoor clear glass and drywall only had 3.6 and $6.8 \mathrm{~dB}$ of loss) [76]. This is why DASs are expected to be important for $5 \mathrm{G}$ in general and $5 \mathrm{G}$ IoT in particular.

3GPP and ETSI propose that the pathloss incorporating O2I building penetration loss be modelled as in the following [81]:

$$
\mathrm{PL}=\mathrm{PL}_{\mathrm{b}}+\mathrm{PL}_{\mathrm{tw}}+\mathrm{PL}_{\mathrm{in}}+N\left(0, \sigma_{P}^{2}\right)
$$

where

$\mathrm{PL}_{\mathrm{b}}$ is the basic outdoor path loss where $d_{3 \mathrm{D}}$ is replaced by $d_{3 \mathrm{D} \text {-out }}+d_{3 \mathrm{D} \text {-in }}$

$\mathrm{PL}_{\mathrm{tw}}$ is the building penetration loss through the external wall,

$\mathrm{PL}_{\text {in }}$ is the inside loss dependent on the depth into the building, and

$\sigma_{P}$ is the standard deviation for the penetration loss

$\mathrm{PL}_{\mathrm{tw}}$ is characterized as:

$$
\mathrm{PL}_{t w}=\mathrm{PL}_{n p i}-10 \log _{10} \sum_{i=1}^{N}\left(p_{i} \times 10^{L_{\text {material } j} /-10}\right)
$$

where

$\mathrm{PL}_{n p i}$ is an additional loss is added to the external wall loss to account for non-perpendicular incidence

$L_{\text {material } i}=a_{\text {material } i}+b_{\text {material } i} \cdot f$, is the penetration loss of material $i$, example values below

$\begin{array}{ll}\text { Material } & \text { Penetration loss }[\mathrm{dB}] \\ \text { Standard multi-pane glass } & L_{\text {glass }}=2+0.2 f \\ \text { IRR glass } & L_{\text {IIRglass }}=23+0.3 f \\ \text { Concrete } & L_{\text {concrete }}=5+4 f \\ \text { Wood } & L_{\text {wood }}=4.85+0.12 f\end{array}$

Note: $\mathrm{f}$ is in $\mathrm{GHz}$

$p_{i}$ is proportion of $i$-th materials, where $\sum_{i=1}^{N} p_{i}=1$; and $N$ is the number of materials.

3GPP TR 38.901 version 14.0.0 Release 14

ETSI TR 138901 V14.0.0 (2017-05)

In consideration of these propagation characteristics, many municipalities in the U.S. are concerned about the possible massive proliferation of small cells needed to support 5G. For example, a filing to the FCC was made in the U.S. late in 2018 by a consortium of towns known as the Communities and Special Districts Coalition in response to the Commission's September 5, 2018, Draft Declaratory Ruling and 3rd Report and Order where the FCC asserted the claim that "small cell" deployment is a federal undertaking; furthermore the filing states that "the massive deployment envisioned by the Commission raises substantial questions as to whether the Commission is in a position to assert that deployment is safe, given that its radio frequency emissions rules were based on technologies and deployment patterns that the Commission declares obsolete in this Order" [74, 91]. Furthermore, it is unclear, according to the filing, what is the size of the equipment needed to support a small cell, since it could vary from a "pizza box" system to several racks that equate to 56 "pizza boxes" [91]. Although small cells will indeed need to be deployed to properly support $5 \mathrm{G}$, caution is advocated. S\&P Global Market Intelligence estimates that small-cell deployments reach approximately 850,000 in the U.S. by 2025 (with approximately 700,000 already deployed in 2019), with about $30 \%$ of small cell installations being outdoors; the same projection forecasts a total of 8.4 million small cells world-wide, with some regions of the world experiencing much higher deployments rates that in the U.S., e.g., doubling the 2019 numbers by the year 2025. These data show that placement within buildings is a common alternative (there will be more in-building systems than outdoor systems) [75].

\section{5G DAS for Indoor IoT Applications}

The previous section discussed propagation issues at the higher frequencies. However, even the sub- $6 \mathrm{GHz}$ bands have issues penetrating buildings with the new building materials and infrared reflecting (IRR) glass. Indoor solutions are needed for IoT even at standard 3G/4G LTE frequencies and much more so at mmWave if cellular-based (5G) IoT transmission services for in-building applications are contemplated; outdoor 5G IoT applications do not.

Although it is in principle possible to support multiple access technologies in an IoT sensor (chipset), end-point IoT devices tend to have low complexity in order to achieve an established target price point and on-board power (battery) budget. Therefore a (large) number of applications will have devices that have a single implemented wireless uplink. It follows that -- either because of the goal of mobility support (for example, a wearable that works seamlessly indoors and in open spaces around town) or because of the designer's goal to utilize a single, consistent IoT nodal and access technology - an all-sites wireless service for a Smart City application, is preferred. DASs may support such a goal (while city-wide WiFi and/or Sigfox/LoRa could be an alternative, the ubiquity, standardization and cost-effectiveness of $5 \mathrm{G}$ cellular and IoT services, may well favor the latter in the future).

4.1. DAS Networks. A DAS is network of a (large) number of (small) (indoor or on-location) antennas connected to a common cellular source via fiber optic channel, providing cellular/wireless service within a given structure. DAS (sometimes also called in-building cellular) refers to the technology that enables the distribution and rebroadcasting of cellular, LTE, AWS, 5G and other RF frequencies within a building or confined/defined structural environment. While DAS is often used in large urban office buildings, DAS can also be used in 
open spaces such as campuses, conference centers, stadiums, hospitals, airports, train stations, tunnels, hotels, cruise ships, and so on. DASs can and will support cellular-based IoT (e.g., LTE-M, NB-IoT, and 5G IoT.) Elements of a DAS include (see Figure 16):

(i) (Small) Broadband antennas and amplifiers in the indoor space (typically one or more per floor) that shape the coverage. These antennas typically cover the entire spectrum of the cellular service (for/from multiple service providers;

(ii) Coax or fiberoptic cabling to connect the structure antennas to a local Base Station;

(iii) Remote Radio Head, a local Base Station, ("small cell"), typically in the basement; and,

(iv) Fiberoptic connection to an aggregation point (typically in a carrier colocation space) (or the use of an outdoor donor antenna to a specific cellular provider). The former supports carrier-neutral applications, the latter typically supports only one carrier. Physical connectivity from the colocation space to each of the wireless providers is needed, typically in the form of fiber connectivity or other telecom service. Business relationships with the wireless providers are needed.

Current typical drivers include the fact that during anticipated peak times (whether in a building or in some public venue as a stadium) users will experience: coverage deficiencies, blocked connections, reduced data speeds, among other service deficiencies. Current systems support CDMA, EVDO, GSM, HSPA, UMTS, among others. Future systems will support $5 \mathrm{G}$ and become even more prevalent.

Given the mmWave transmission issues mentioned above (the small cells, the directionality, the free space loss and other attenuation factors) DASs will likely play a big role in $5 \mathrm{G}$, both for regular voice and data services and for IoT. The large number of "small cells" cited earlier (8.4 million in 2025 , with about $70 \%$ of these being considered to be indoors) supports the thesis that DASs will play a pivotal role in the future. They will be a key element of Smart City IoT support, especially for in-building sensors. As was shown in Figure 2, while a number of applications could use a Wi-Fi (or related) access technology with networked connection to the cloud, or a Sigfox/LoRa related solutions (these, however, being vendor proprietary), Smart City IoT service implementers may prefer to use a cellular service such as LTE-M or NB-IoT in the immediate future and 5G IoT as it becomes available, allowing a seamless and single-technology solution city-wide. In some cases, for example in smaller/older buildings and/or in suburbia and/or for buildings very close to a $5 \mathrm{G}$ cell tower, a direct $5 \mathrm{G}$ IoT connection may suffice. But for high-density urban and smart building applications, the use of DASs seems inevitable.

4.2. DAS Design. A single, carrier-neutral, consolidated system is often sought: a carrier-neutral system avoids multiplicity of antenna distribution, and sharing allows more coverage and higher capacity. A carrier-neutral DAS supports an end-use system, for example a smartphone, regardless of which service provider the user is subscribed to. It would be rather expensive for a building owner to deploy a carrierneutral DAS that supports a single building, unless it would be a very large building, campus, or installation. With carrier-neutral DAS arrangements the ownership of system is shifted from the building owner, or a specific cellular carrier to a third-party system provider, or a DAS integrator. Figure 17 depicts a typical carrier-neutral arrangement. Obtaining wireless carrier permission and coordinating between different wireless carriers is a key planning undertaking of any successful DAS rollout. Three scenarios are shown:

(i) Scenario/Approach S1: The DAS integrator/provider wires up a remote building or space and drops a fiber link into an existing colo rack at an existing carrierneutral provider, thus sharing all the Base Station Hotel (BSH) colo equipment and interfaces to the various wireless providers.

(ii) Scenario/Approach S2: The DAS integrator/provider must build out the requisite base station equipment in the colo (the colo provider only provides power, rack space, HVAC, and so on). The DAS integrator/provider must also build interfaces to the wireless providers and secure business arrangements with them. The DAS integrator/provider builds out the remote buildings or venues.

(iii) Scenario/Approach S3: The DAS integrator/provider must build out the requisite base station equipment in the colo, but the DAS integrator/provider can make use of existing interfaces and equipment to the various wireless providers. The DAS integrator/provider builds out the remote buildings or venues.

A less desirable approach is to use "donor antennas" (also shown in Figures 16 and 17). These antennas are installed on the roof of a building and are pointed at "donor" cell towers. Typically, a single cellular vendor is supported. The in-building arrangement is similar to that of a carrier-neutral arrangement, except that there typically will not be a remote base station: a combination of fiber optic cable, coaxial cable and in-building antennas is used to amplify and distribute those signals within a given space; coordination with the given carrier is still needed to make sure that the concentrated traffic is accepted by the provider.

\section{5G Deployment Snapshot}

5.1. 5 G Cellular Services. According to GSMA 5G is on track to account for $15 \%$ (1.4 billion) of global mobile connections by 2025 . By early 2019, according to GSMA, eleven worldwide operators had announced initial 5G service launches and seven other operators had activated $5 \mathrm{G}$ base stations with commercial services to follow in the near future [92]. Select cities worldwide will have 5G by the end of 2019. See Table 6 for a summary of near-term $5 \mathrm{G}$ service-deployment activities. However, $4 \mathrm{G}$ services are expected to continue well past 2025: $4 \mathrm{G}$ will account for $59 \%$ of the connections, $3 \mathrm{G}$ for $20 \%$ of the connections, and $2 \mathrm{G} 5 \%$ of the connection $(3 \mathrm{G}$ and $2 \mathrm{G}$ 


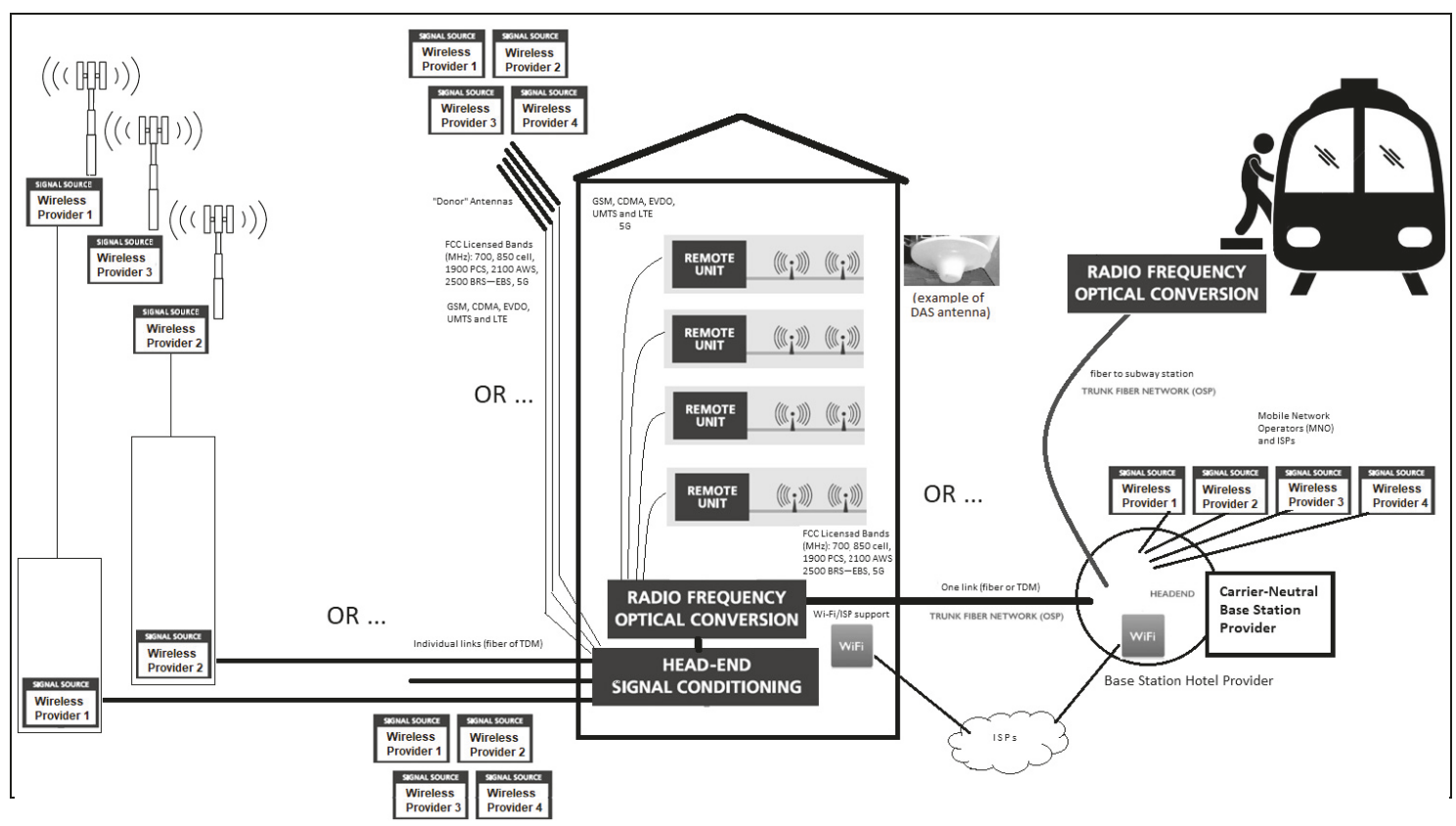

Figure 16: Elements of a DAS.

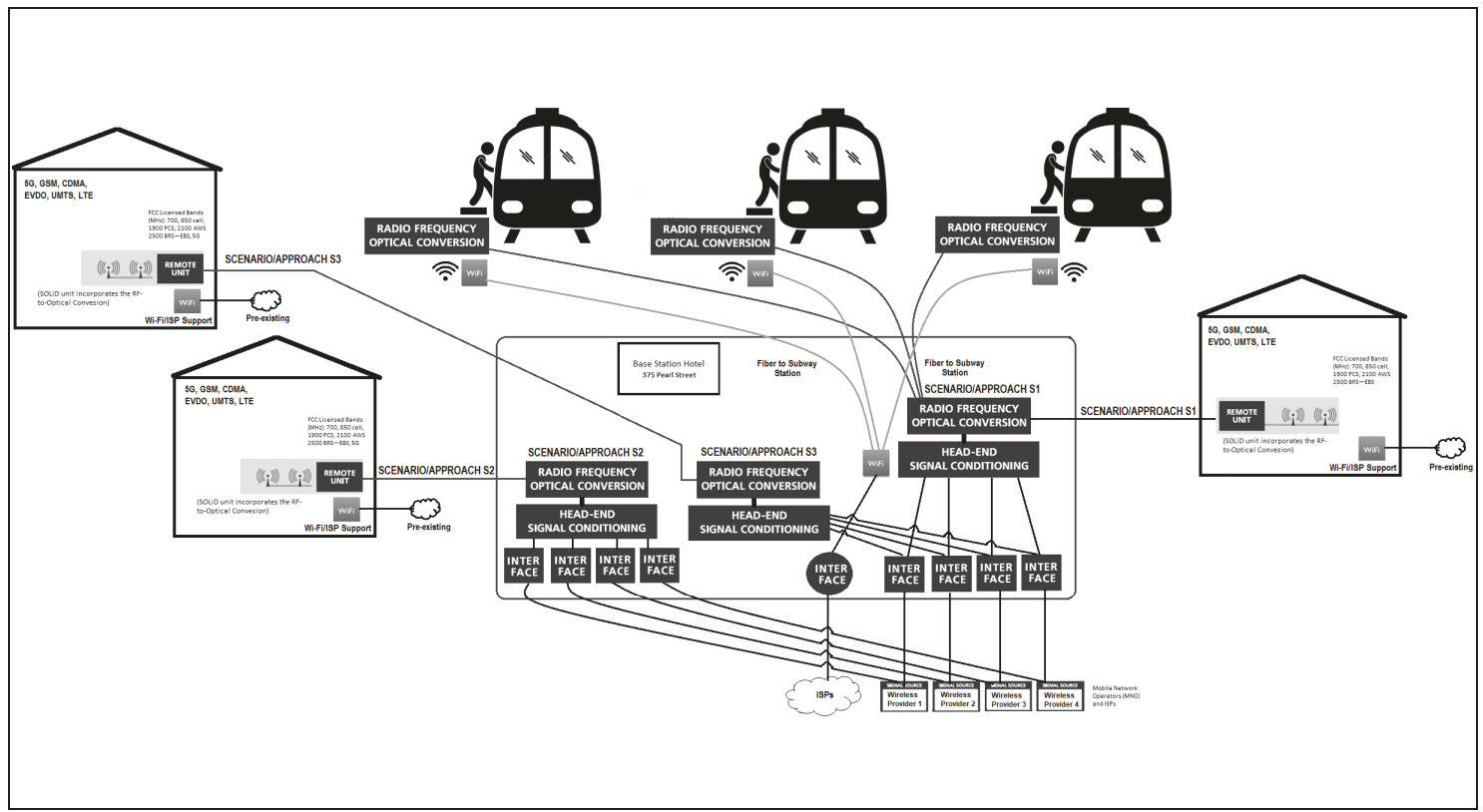

Figure 17: Carrier-neutral DAS.

are trending down through this period while $4 \mathrm{G}$ will continue to grow, but settle at around $60 \%$ by $2023--5 \mathrm{G}$ is penetrating at approximate linear rate of CAGR around 1.5-2\%). Also, according to GSMA 2019 will see 5G launches accelerate and devices hit the market:16 major markets worldwide will start to offer commercial $5 \mathrm{G}$ networks in 2019, following on from the first 5G launches in South Korea and the U.S. in 2018, as follows [28]:

(i) Q4 2018: South Korea, U.S. (ii) Q1 2019: Bahrain, Czech Republic, Estonia, Finland, Saudi Arabia, Switzerland

(iii) Q2 2019: Australia, Qatar

(iv) Q3 2019: Austria, China, Hong Kong, Kuwait, Spain, UAE

(v) Q4 2019: Portugal, UK

As of Q2 2019, there were 303 rollouts of 5G mobile networks across 294 locations worldwide, operated by 20 mobile 
TABLE 6: Summary of near-term 5G service-deployment activities (2019 view).

\begin{tabular}{|c|c|}
\hline Country or Region & Near-term 5G Activities \\
\hline South Korea & $\begin{array}{l}\text { Korea Telecom rolled out a trial 5G network in support of the } 2018 \text { Winter Olympics in Seoul, } \\
\text { South Korea covering events in several cities. It has also launched a VR/AR games platform } \\
\text { supported from the cloud over 5G. SK Telecom has acquired spectrum in the } 3.5 \mathrm{GHz} \text { and } 28 \mathrm{GHz} \\
\text { frequencies in preparation of deploying } 5 \mathrm{G} \text {. }\end{array}$ \\
\hline China & $\begin{array}{l}\text { China plans early implementations of 5G. The GSMA estimates that by } 2025 \text { China will represent } \\
40 \text { percent of global 5G connections. According to the GSMA, with } 460 \text { million projected users } \\
\text { China is expected to become the world's largest 5G market by 2025, higher than Europe's } 205 \\
\text { million and the United States' } 187 \text { million combined. China's three major mobile operators - } \\
\text { China Mobile, China Unicom and China Telecom - are rolling out trial operations of 5G systems } \\
\text { in several cities, and all three aim to fully commercialize the technology by } 2020 \text { [66]. }\end{array}$ \\
\hline India & 5G trials are contemplated by late 2019 and early deployments may happen late in 2020. \\
\hline Japan & $\begin{array}{l}\text { NTT DoCoMo demonstrated an advanced security service based on 5G network technology for } \\
\text { use in the } 2020 \text { Olympics. }\end{array}$ \\
\hline United States & $\begin{array}{l}\text { Migration from the } 4 \mathrm{G} \text { networks should be relatively simple. The FCC has been making several } \\
\text { new bands available as noted elsewhere. Verizon has been aggressive in its advertisement } \\
\text { campaigns about its introduction of } 5 \mathrm{G}-\text { related services. }\end{array}$ \\
\hline Europe & $\begin{array}{l}\text { T-Mobile is preparing for the rollout of } 5 \mathrm{G} \text { in } 2020 \text {, starting in the Netherlands. Some fear that } \\
\text { Europe risks falling behind other regions because of restrictive regulation and weak investments; } \\
\text { less than half of the countries in Europe have actually allocated spectrum for 5G [67]. }\end{array}$ \\
\hline
\end{tabular}

carriers [93]. In the U.S., 21 deployments were documented, of which five were in Texas, four in California, two each in North Carolina and Florida, and one each in Oklahoma, Minnesota, Illinois, Indiana, Kentucky, Tennessee, Georgia, and Louisiana (some of these, such as the AT\&T 5G network in Louisville, KY, had "Limited Availability" at that time.)

5.2. MmWave Spectrum. Regarding frequency bands 3GPP is initially focusing on $24 \mathrm{GHz}$ to $43 \mathrm{GHz}$ mmWave spectrum (Release 15.) Other ongoing 5G work relates to NSA and SA configurations Massive MIMO, beamforming, and LTE interoperability. 3GPP Release 16 (2019) aims at full compliance to IMT-2020 (e.g., supporting $1 \mathrm{GHz}$ channels) and other spectrum capabilities (e.g., spectrum sharing, additional bands, and URLCC).

In the U.S., among other possible candidates, the FCC is making available new frequency bands for $5 \mathrm{G}$ use under its rubric of "Spectrum Frontiers proceeding", of which three have been instituted in the recent past. With the "July 2016 Order", the FCC designated the 27.5-28.35 GHz (known as the "28 GHz band"), 37-38.6 GHz (known as the "37 $\mathrm{GHz}$ band"), and 38.6-40 GHz (known as the "39 GHz band") bands for flexible mobile and fixed commercial use, and designated the 64-71 GHz band for unlicensed use (to supplement 57-64 GHz which had been made available for unlicensed use at an earlier time.) While the FCC has yet to auction any of the new Upper Microwave Flexible Use Service or (UMFUS) spectrum, in 2017, with a Second Report and Order, a Memorandum Opinion and Order it designated an additional 1700 megahertz of mmWave spectrum for licensed flexible commercial wireless fixed and mobile use. The 1700 $\mathrm{MHz}$ spectrum covered the 24.25-24.45, 24.75-25.25, and 47.2-48.2 GHz bands (the first two known collectively as the "24 GHz" bands, and the third, known as the "47 $\mathrm{GHz}$ " band). Therefore, the spectrum at $24.25-24.45 \mathrm{GHz}$ is now allocated for non-Federal fixed and mobile services on a co-primary basis, and the spectrum at $24.75-25.25 \mathrm{GHz}$ for non-Federal fixed, mobile, and fixed-satellite (FSS) services on a co-primary basis [94].

In terms of rollouts, in the U.S, the spectra at 27.5 28.35 GHz and $37-40 \mathrm{GHz}$ may see preliminary commercial deployments in 2019; in Korea, the spectrum at 26.5 - 29.5 $\mathrm{GHz}$ is similarly expected to see commercial deployments in 2019; and the EU expects commercial deployments for the 24.25 - $27.5 \mathrm{GHz}$ spectrum starting around 2020 .

In addition to the radio access for the end-user device, there is also interest in Backhaul and now also in Fronthaul. Backhaul mechanisms are mechanisms to connects the wireless network to the wired network by backhauling traffic from dispersed cell sites to Mobile Switching Offices (MSOs). These links typically are either traditional transmission systems (such as SONET or point-to-point microwave at various operating bands), or they are Ethernet-over-Fiber links (e.g., $1 \mathrm{GbE}$ or $10 \mathrm{GbE})$. A UMa site has Baseband Unit (BBU) that processes user and control data, which is in turn connected to a Radio Unit (RU) to generate radio signals transmitted over the air via the tower-mounted antennas.

Fronthaul is related to a new type of Radio Access Network (RAN) architecture that is comprised of centralized baseband controllers and standalone radio heads installed at remote UMa or UMi sites possibly many miles away. In the fronthaul model the BBU and RU equipment is located further away from each other than is the case in the backhaul model. The RU equipment (now referred to as a Remote Radio Head [RRH]) is still located at the cell site, but the BBU is relocated to centralized location where it supports multiple RRHs. See Figure 18. The optical links that interconnect the newly centralized BBU and the multiple RRHs is are referred to as fronthaul. The use of fronthaul-based C-RAN (Cloud-RAN) architectures typically improves the cell edge performance. Backhaul and fronthaul are key use cases for mmWave spectrum and will play a role in 5G and 5G IoT. 


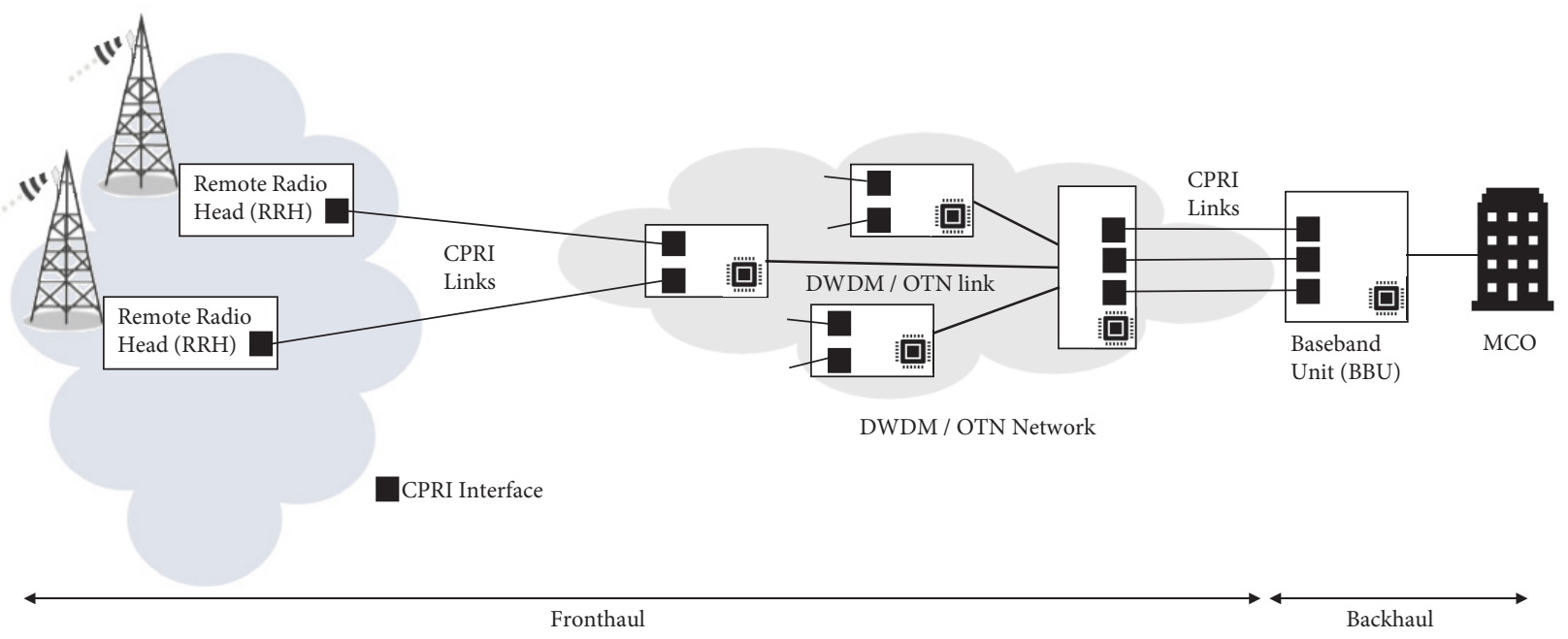

FIGURE 18: Fronthaul and Backhaul.

A consortium of wireless equipment vendors standardized the Common Public Radio Interface (CPRI) protocol that runs over these fronthaul links a few years ago; more recently, a newer eCPRI 1.0 interface has been defined; additionally, work is underway to defining a more detailed interface. The tight performance requirements of CPRI/eCPRI -- capacity, distance, and latency - drive towards fiber connectivity such as DWDM (or more specifically OTN [Optical Transport Network]) systems between centralized BBUs and the RRHs. Ethernet-based solutions have existed for a number of years using mmWave spectrum. Work is underway in 3GPP to define backhauling solutions using the same spectrum as access. Work is also underway to define new fronthaul interfaces also utilizing mmWave spectrum.

5.3. 5G IoT Services. Global IoT revenue are expected to increase at an annual rate of $23 \%$ to 2025 to reach $\$ 1.1$ trillion (up from 267 B in 2018). As discussed in the next section, near term " $5 \mathrm{G}$ IoT" really equate to NB-IoT and LTE-M capabilities. At the end of 2018, there were 83 commercial deployments of LTE-M and NB-IoT worldwide. However, pure connectivity will become increasingly commoditized, making it difficult for operators to compete on the data transmission alone, declining from $9 \%$ of total IoT revenue in 2018 to $5 \%$ in 2025. Service providers must develop new strategies and business models beyond connectivity services. Applications, platforms and services (e.g., cloud data analytics and IoT security) are the major growth areas of IoT; this segment will be approximately $70 \%$ of the market in 2025 . Professional services (e.g., consulting, systems integration, also including managed services) will increase in share and will be approximately $25 \%$ of the market in 2025 [28].

\section{Current Alternatives and Convergence to 5G}

5G IoT will need to compete with other technologies, both of the cellular type (e.g., NB-IoT and LTE-M) as well as the non-cellular type (although NB-IoT and LTE-M are now considered "part of the $5 G$ world"). The economics and availability of these "legacy" networks in various parts of the world may be such that a level of inertia, frustrating a full migration to truly-novel 5G IoT services, will take hold. Clearly, in principle, $5 \mathrm{G}$ is better positioned for city/regionwide applications as contrasted with building or campus applications.

From an end-user perspective, design and implementation questions center around the following issues, which $5 \mathrm{G}$ IoT technology must be able to address successfully:

(i) Availability of equipment;

(ii) Availability of service (geographic coverage in the area of interest);

(iii) Support of required technical details (latency, bandwidth, packet loss, and so on);

(iv) Support of mobility (where needed, e.g., wearables, crowdsensing, Vehicle to Vehicle and Vehicle to Infrastructure applications, to name a few);

(v) Adequate reliability (where needed, e.g., physical security, process control, Vehicle to Vehicle and Vehicle to Infrastructure applications, to name a few);

(vi) Scalability support (functional and geographic/numerical expansion of the application)

(vii) Initial and recurring cost of the equipment; and

(viii) Initial and recurring cost of the service.

Recent acceptability and economics of NB-IoT and LTE-M can serve as a proxy for the near-term commercial success of $5 \mathrm{G}$ IoT in particular and truly-novel 5G IoT services in general. Some developers have looked at cellular services for city-wide or region-wide IoT coverage; in some instances, for example, for national truck transportation a combination of Low Earth Orbit (LEO) satellite service and cellular services have and are being used. A current drawback is the cost of the requisite (miniaturized) modems and the cost of the cellular service. New services such as NB-IoT and LTE CatM1 (an LTE-based 3GPP-sponsored alternative to NB-IoT, 
also known as LTE-M) are short term attempts to address the cost and resource issues. In particular, NB-IoT is seen as providing a pathway to 5G IoT. 5G and truly-novel 5G IoT are the target solutions.

6.1. NB-IoT. As noted earlier, NB-IoT is a licensed low power LPWAN technology designed to coexist with existing LTE specifications and providing cellular-level QoS connectivity for IoT devices. NB-IoT was standardized by 3GPP in LTE Release 13, but it does not operate in the LTE context per se [95-97]. NB-IoT has attracted support from Qualcomm, Ericsson and Huawei, among many other vendors and service providers. NB-IoT (also known as LTE Cat-NB1) is based on a Direct Sequence Spread Spectrum (DSSS) modulation in a $200 \mathrm{kHz}$ channel. There are several underutilized $200-\mathrm{kHz}$ GSM spectrum channels, as well as other possible bands such as guard bands. NB-IoT is intended as an alternative to LoRa and Sigfox. This technology can optimize sunken financial investments by service providers and can shorten the service deployment rollout timetable for IoT services, since NB-IoT uses existing cellular infrastructure. NB-IoT service goals include: (i) low complexity end-nodes, (ii) device cost less than $\$ 5$, (iii) a device battery life expected to last for 10 years if it transmits 200 bytes of data per day, and (iv) uplink latency less than $10 \mathrm{~s}$ (thus not a true real-time service). NB-IoT operates on $900-1800 \mathrm{MHz}$ frequency bands with coverage of up to about 20 miles; it supports data rates of up to 250 Kbps for uplink and $230 \mathrm{Kbps}$ for downlink communications [98-101]. NB-IoT can be implemented in a number of ways: (i) in standalone non-cellular licensed bands; (ii) in unused $200 \mathrm{kHz}$ bands in the context of GSM or CDMA; and (iii) in LTE environments where base stations can allocate a resource block to NB-IoT transmissions. Since NB-IoT offers low cost for the device and for the service, it is a good choice for largescale distributed deployment in Smart Cities and smart grid applications.

As illustrative commercial examples, in 2018 T-Mobile announced a North American NB-IoT plan that costs just $\$ 6$ a year - one tenth of Verizon's Cat-M plans- for up to $12 \mathrm{MB}$ per connected device, and several NB-IoT modules based on Qualcomm ${ }^{\circledR}$ MDM9206 LTE IoT modem that are certified for use on T-Mobile's network. T-Mobile, in conjunction with Qualcomm and Ericsson conducted the first trial NB-IoT in the U.S. in 2017 across multiple sites; T-Mobile and the City of Las Vegas also announced a partnership to deploy IoT technology throughout the city. For applications that require more bandwidth and voice, T-Mobile offers Cat-1 IoT Access Packs $[102,103]$. NB-IoT consumes minimal power: while most IoT end-nodes save power when they are quiescent, when the node and the modem are running and handling all the signal processing, the systems with simpler waveform (such NB-IoT) consume less overall power. Additionally, chipsets that support a single protocol (such as NB-IoT) are cheaper compared to a chipset that supports multiple protocols. Furthermore, prima facie, NB-IoT may provide deeper building penetration than LTE-M.

6.2. LTE-M. LTE-M is a power-efficient system, where two innovations support battery efficiency: LTE eDRX (Extended
Discontinuous Reception) and LTE PSM (Power Saving Mode). LTE-M allows the upload of 10 bytes of data a day (LTE-M messages are fairly short compared to NBIoT messages), but also allows access to Mbps rates. Therefore, LTE-M can support several use cases. In the U.S., major carriers such as Verizon and AT\&T offer LTE-M services (as noted, Verizon has announced support for NBIoT -- T-Mobile and Sprint appears to lean in the NBIoT direction) [104]. Worldwide geographies with GSM deployments will likely offer NB-IoT in the short term. Figure 19 depicts some of the IoT compatibility mechanisms to be incorporated into $5 \mathrm{G}$ in terms of band and bandwidth; however, the transmission frequencies will be wildly different.

In summary, LTE-M supports low nodal complexity, high nodal density, low nodal power consumption, low latency and extended geographic coverage, while allowing service operators the reuse of the LTE installed base. NBIoT aims at improved indoor coverage, high nodal density for low throughput devices, low delay sensitivity, low node cost, low nodal power consumption and simplified network architecture. NB-IoT and LTE-M are currently providing mobile IoT solutions for smart cities, smart logistics, and smart metering, but only in small deployments to date (as of early 2018, there were 43 commercial NB-IoT and LTE-M networks worldwide [105]). As noted, the commercial success of NB-IoT and LTE-M can serve as a proxy for the eventual success of $5 \mathrm{G}$ IoT in a smart city context (compared with noncellular LPWAN solutions).

NB-IoT, LTE-M and LTE are 4G standards, but advocates claim that they remain integral parts of early releases of 5G. Proponents make the case that "enterprises deploying either NB-IoT or LTE-M are futureproofing their IoT projects because when $5 G$ rollouts become commonplace these two Mobile IoT standards will continue into foreseeable $5 G$ releases (from 3GPP Release 15 on)" [102]. In the context of 3GPP Rel 15, it appears, in fact, that NB-IoT and LTE-M will be included as 5G mobile standards. In 2018 the GSMA asserted that "NB-IoT and LTE-M, as deployed today, are part of the $5 G$ family; with the dawn of the $5 G$ era [...] both $N B$ IoT and LTE-M technologies are an integral part of 5G, and that $5 G$ from the LPWA perspective, is already here today" [105]. Including these technologies as initial 5G IoT standards will motivate service providers and vendors to support these implementations for IoT deployments as an evolutionary strategy to 5G. 3GPP Release 16 (targeted for the end of 2019) is considered to be the "second 5G standard" and thereafter transmitted to the ITU for consideration as a global standard. Among other functionality and capabilities, Release 16 is expected to add standards for connected cars and smart factories (notably, automobile companies have formed the $5 \mathrm{G}$ Automotive Association to assist 3GPP to set autonomous vehicle standards, such as $5 \mathrm{G}$ cellular vehicle-to-everything [C-V2X]).

\section{Conclusion}

This paper discussed a number of issues related to 5G-based IoT applications, particularly in Smart Cities environments, 


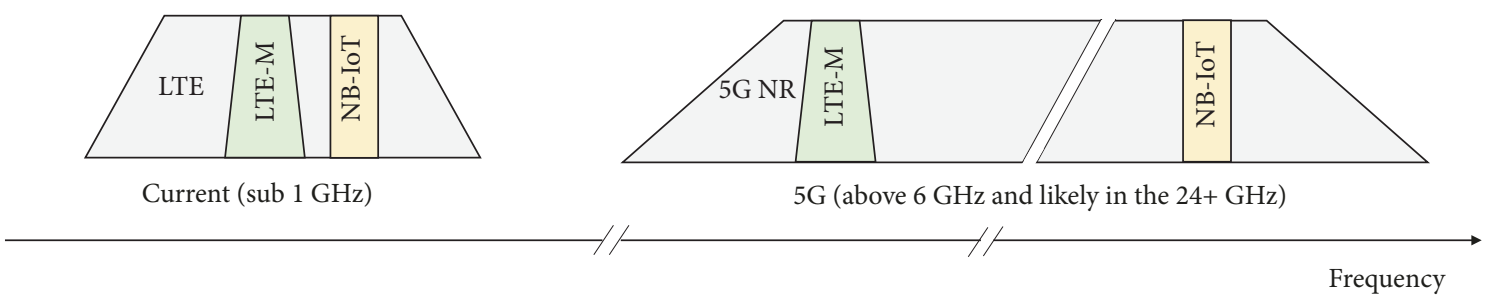

FIgURE 19: Support of LTE-M and NB-IoT under 5G.

including the need for small cells, the transmission issues at the millimeter wave frequencies, building penetration issues, the need for DAS, and the near term introduction of pre-5G IoT technologies such as NB-IoT and LTE-M, these being possible proxies for $5 \mathrm{G}$ IoT deployment.

A firm definition of 5G IoT has still to emerge, although a large number of use cases have been described by various industry entities. Both 3GPP NB-IoT and LTE-M technologies are seen at this juncture as integral to $5 \mathrm{G}$ services: these $4 \mathrm{G}$ technologies are expected to continue under full support in $5 \mathrm{G}$ networks for the immediate future. However, IoT/Smart City applications that require high bandwidth will need implementations of eMBB and mmWave frequencies.

Some controversy existed at press time about the development of $5 \mathrm{G}$ equipment, in the context of origin-ofmanufacturing and the possible intrinsic risk related to cybersecurity [106]. If these issues are not satisfactorily resolved, some delay in the broad early deployment of 5G may result. However, the expectation is that these issues will work themselves out over time.

\section{Conflicts of Interest}

The authors declare that they have no conflicts of interest.

\section{References}

[1] National League of Cities and Center for City Solutions and Applied Research, "City of the future - technology and mobility," White Paper 1301, Pennsylvania Avenue, Suite 550, Washington, DC, USA, 2015.

[2] A. Ramaswami, A. G. Russell, P. J. Culligan, K. Rahul Sharma, and E. Kumar, "Meta-principles for developing smart, sustainable, and healthy cities," Science, vol. 352, no. 6288, pp. 940-943, 2016.

[3] D. R. Martínez, T. J. Gracia, E. M. Muñoz, and A. C. García, "Smart cities' challenge: how to improve coordination in the supply chain," in Sustainable Smart Cities, Innovation, Technology, and Knowledge Management, pp. 129-142, Springer International Publishing, 2016.

[4] N. Mali, "A review on smart city through internet of things (IoT)," International Journal of Advanced Research in Science Management and Technology, vol. 2, no. 6, 2016.

[5] A. Caragliu, C. D. Bo, and P. Nijkamp, "Smart cities in europe," Journal of Urban Technology, vol. 18, no. 2, pp. 65-82, 2011 (Chinese).

[6] D. Minoli and B. Occhiogrosso, "Internet of things applications for smart cities," in Internet of Things A to Z: Technologies and
Applications, Q. Hassan, Ed., Chapter 12, IEEE Press/Wiley, 2018.

[7] A. Zanella, N. Bui, A. P. Castellani, L. Vangelista, and M. Zorzi, "Internet of things for smart cities," IEEE Internet of Things Journal, vol. 1, no. 1, pp. 22-32, 2014.

[8] D. Minoli and B. Occhiogrosso, "Mobile IPv6 protocols and high efficiency video coding for smart city IoT applications," in Proceedings of the 13th International Conference and Expo on Emerging Technologies for a Smarter World (CEWIT), pp. 1-6, Stony Brook, New York, NY, USA, 2017.

[9] B. J. Wewalaarachchi, H. Shivanan, and H. Gunasingham, "Integration platform to enable operational intelligence and user journeys for smart cities and the internet of things," in Proceedings of the Patent US20160239767 A1, 2016.

[10] S. Srivastava and N. Pal, "Smart cities: the support for internet of things (IoT)," International Journal of Computer Applications in Engineering Sciences, pp. 5-7, 2016.

[11] O. Bates and A. Friday, "Beyond data in the smart city: repurposing existing campus IoT," IEEE Pervasive Computing, vol. 16, no. 2, pp. 54-60, 2017.

[12] D. Kyriazis, T. Varvarigou, D. White et al., "Sustainable smart city IoT applications: heat and electricity management \& ecoconscious cruise control for public transportation," in Proceedings of the IEEE 14th International Symposium on "A World of Wireless, Mobile and Multimedia Networks" (WoWMoM), IEEE, Madrid, Spain, 2013.

[13] D. Minoli and B. Occhiogrosso, "IoT applications to smart campuses and a case study," European Union Digital Library, vol. 5, article e4, pp. 2518-3893, 2017.

[14] A. Al-Fuqaha, M. Guizani, M. Mohammadi et al., "Internet of things: a survey on enabling technologies, protocols, and applications," IEEE Communication Surveys \&Tutorials, vol. 17, no. 4, pp. 2347-2376, 2015.

[15] R. Gomes, H. Pombeiro, C. Silva et al., "Towards a smart campus: building-user learning interaction for energy efficiency, the lisbon case study," in Handbook of Theory and Practice of Sustainable Development in Higher Education, World Sustainability Series, pp. 381-398, Springer, 2016.

[16] Z. Yu, Y. Liang, B. Xu et al., "Towards a smart campus with mobile social networking," in Proceedings of the 4th IEEE Int'l Conference on Cyber, Physical and Social Computing (CPSCom), pp. 162-169, IEEE, Dalian, China, 2011.

[17] A. Roy, J. Siddiquee, A. Datta et al., "Smart traffic \& parking management using IoT," in Proceedings of the IEEE 7th Annual Information Technology, Electronics and Mobile Communication Conference (IEMCON), IEEE, Vancouver, BC, Canada, 2016.

[18] R. Grodi, D. B. Rawat, and F. Rios-Gutierrez, "Smart parking: Parking occupancy monitoring and visualization system for 
smart cities," in Proceedings of the SoutheastCon, IEEE, Norfolk, VA, USA, 2016.

[19] D. Minoli, K. Sohraby, and B. Occhiogrosso, "IoT considerations, requirements, and architectures for smart buildings energy optimization and next generation building management systems," IEEE Internet of Things Journal, vol. 4, no. 1, pp. 269283, 2017.

[20] L. Kang, S. Poslad, W. Wang et al., "A public transport bus as a flexible mobile smart environment sensing platform for IoT," in Proceedings of the 12th International Conference on Intelligent Environments (IE), IEEE, London, UK, 2016.

[21] M. Alam, J. Ferreira, and J. Fonseca, "Introduction to intelligent transportation systems," in Journal of Intelligent Transportation Systems, vol. 52 of Studies in Systems, Decision and Control, pp. 1-17, Springer International Publishing, 2016.

[22] J. Barbaresso, G. Cordahi, and et al, "The national academies of science, engineering and medicine, USDOT's intelligent transportation systems (ITS): its strategic plan," The National Academies of Science, Engineering and Medicine, USDOT's Intelligent Transportation Systems (ITS): ITS Strategic Plan 2015-2019 FHWA-JPO-14-145, 2014.

[23] S. H. Sutar, R. Koul, and R. Suryavanshi, "Integration of Smart Phone and IOT for development of smart public transportation system," in Proceedings of the International Conference on Internet of Things and Applications (IOTA), pp. 73-78, Pune, India, 2016.

[24] M. Picone, S. Busanelli, M. Amoretti et al., Advanced Technologies for Intelligent Transportation Systems, Springer, 2015.

[25] Q. Wang, Y. Zhao, W. Wang et al., "Multimedia IoT systems and applications," in Proceedings of the Global Internet of Things Summit (GIoTS), IEEE, Geneva, Switzerland, 2017.

[26] D. Minoli and B. Occhiogrosso, "Ultrawideband (UWB) technology for smart cities IoT applications," in Proceedings of the IEEE International Smart Cities Conference (ISC2), pp. 1-8, IEEE, Kansas City, Mo, USA, 2018.

[27] R. K. Ganti, F. Ye, and H. Lei, "Mobile crowdsensing: current state and future challenges," IEEE Communications Magazine, vol. 49, no. 11, pp. 32-39, 2011.

[28] Global System for Mobile Communications Association (GSMA), The Mobile Economy 2019, 2019, https://www.gsmaintelligence.com/research/?file=b9a6e6202eeld5f787cfebb95d3639c5\&amp; download.

[29] Statistica, Internet of things (IoT) connected devices installed base worldwide from 2015 to 2025 (in billions), 2015, https://www .statista.com/statistics/471264/iot-number-of-connected-devices-worldwide/.

[30] "Recommendation ITU-R M.2083-0. IMT vision-framework and overall objectives of the future development of IMT for 2020 and beyond," http://www.itu.int/rec/R-REC-M.2083-0201509-I/.

[31] "Next generation mobile networks," 5G White Paper, 2015, https://www.ngmn.org/fileadmin/ngmn/content/downloads/ Technical/2015/NGMN_5G_White_Paper_V1_0.pdf.

[32] "Feasibility study on new services and markets technology enablers," 3GPP 22.891, 2019, http://portal.3gpp.org/desktopmodules/Specifications/SpecificationDetails.aspx?specificationId=2897.

[33] H. Yu, H. Lee, and H. Jeon, "What is 5G? emerging $5 \mathrm{G}$ mobile services and network requirements," Sustainability, vol. 9, no. 10, article 1848, 2017.
[34] GSMA, Road to 5G: introduction and migration, 2018, https:// www.gsma.com/futurenetworks/wp-content/uploads/2018/04/ Road-to-5G-Introduction-and-Migration_FINAL.pdf.

[35] S. W. Hu and C. M. Shy, "Health effects of waste incineration: a review of epidemiologic studies," Journal of the Air \& Waste Management Association, vol. 51, no. 7, pp. 1100-1109, 2001.

[36] A. Santarsiero, G. Trevisan, G. Cappiello et al., "Urban crematoria emissions as they stand with current practice," Microchemical Journal, vol. 79, no. 1-2, pp. 299-306, 2005.

[37] M. Takaoka, K. Oshita, N. Takeda, and S. Morisawa, "Mercury emission from crematories in Japan," Atmospheric Chemistry and Physics, vol. 10, no. 8, pp. 3665-3671, 2010.

[38] N. Takeda, M. Takaoka, K. Oshita, and S. Eguchi, "PCDD/DF and co-planar PCB emissions from crematories in Japan," Chemosphere, vol. 98, pp. 91-98, 2014.

[39] Y. Xue, H. Tian, J. Yan et al., "Present and future emissions of HAPs from crematories in China," Atmospheric Environment, vol. 124, pp. 28-36, 2016.

[40] D. Muenhor, J. Satayavivad, W. Limpaseni et al., "Mercury contamination and potential impacts from municipal waste incinerator on Samui Island, Thailand," Journal of Environmental Science and Health, Part A: Toxic/Hazardous Substances and Environmental Engineering, vol. 44, no. 4, pp. 376-387, 2009.

[41] S. Sakai, K. Hayakawa, H. Takatsuki, and I. Kawakami, "Dioxinlike PCBs released from waste incineration and their deposition flux," Environmental Science \& Technology, vol. 35, no. 18, pp. 3601-3607, 2001.

[42] G. D. Hinshaw and A. R. Trenholm, "Hazardous waste incineration emissions in perspective," Waste Management, vol. 21, no. 5, pp. 471-475, 2001.

[43] D. C. Ashworth, G. W. Fuller, M. B. Toledano et al., "Comparative assessment of particulate air pollution exposure from municipal solid waste incinerator emissions," International Journal of Environmental Research and Public Health, vol. 2013, 13 pages, 2013.

[44] Chapter 4 in Waste Incineration \& Public Health, National Research Council (US) Committee on Health Effects of Waste Incineration. Washington (DC): National Academies Press (US), 2000, ISBN-10: 0-309-06371-X. Also at https://www.ncbi.nlm .nih.gov/books/NBK233615/.

[45] S. Bose-O'Reilly, K. M. McCarty, N. Steckling et al., "Mercury exposure and children's health," Current Problems in Pediatric and Adolescent Health Care, vol. 40, no. 8, pp. 186-215, 2010.

[46] G. González-Cardoso, N. Santiago, J. M. Hernández-Contreras, and M. Gutiérrez, "PM2.5 emissions from urban crematoriums," Energy Procedia, vol. 153, pp. 359-363, 2018.

[47] METIS, mobile and wireless communications enablers for the twenty-twenty (2020) Information society, the 5G future scenarios identified by METIS -the first step toward A 5G mobile and wireless communications system, 2013.

[48] A. Osseiran, V. Braun, T. Hidekazu et al., "The foundation of the mobile and wireless communications system for 2020 and beyond: challenges, enablers and technology solutions," in Proceedings of the IEEE 77th Vehicular Technology Conference (VTC Spring), IEEE, Dresden, Germany, 2013.

[49] ICT-317669 METIS project, "Requirements and general design principles for new air interface," https://www.metis2020.com/ documents/deliverables/, 2013.

[50] ICT-317669 METIS project, "Positioning of multi-node/multiantenna transmission technologies," https://www.metis2020 .com/documents/deliverables/, 2013. 
[51] ICT-317669 METIS project, "Intermediate description of the spectrum needs and usage principles," https://www.metis2020 .com/documents/deliverables/, 2013.

[52] ICT-31766 METIS project, "Summary on preliminary trade-off investigations and first set of potential network-level solutions," https://www.metis2020.com/documents/deliverables/, 2013.

[53] ICT-317669 METIS project, "Novel radio link concepts and state of the art analysis," https://www.metis2020.com/ documents/deliverables/, 2013.

[54] ICT-317669 METIS project, "Components of a new air interface - building blocks and performance," https://www.metis2020 .com/documents/deliverables/, 2014.

[55] "Small cell forum, mmWave 5G eMBB use cases \& small cell based hyperdense networks," 2017.

[56] X. Ge, L. Pan, Q. Li et al., "Multipath cooperative communications networks for augmented and virtual reality transmission," IEEE Transactions on Multimedia, vol. 19, no. 10, pp. 2345-2358, 2017.

[57] G. Tech, Y. Chen, K. Müller et al., "Overview of the multiview and $3 \mathrm{D}$ extensions of high efficiency video coding," IEEE Transactions on Circuits and Systems for Video Technology, vol. 26, no. 1, pp. 35-49, 2016.

[58] J. Horwitz, "FCC expands $3.5 \mathrm{GHz}$ band to $5 \mathrm{G}$ and opens $6 \mathrm{GHz}$ band to future Wi-Fi," https://venturebeat.com/2018/10/23/ fcc-expands-3-5ghz-band-to-5g-and-opens-6ghz-band-to-future-wi-fi/, 2018.

[59] D. Minoli, K. Sohraby, and B. Occhiogrosso, "IoT security (IoTSec) mechanisms for e-health and ambient assisted living applications," in Proceedings of the 2nd IEEE International Conference on Connected Health: Applications, Systems and Engineering Technologies (CHASE), IEEE, Philadelphia, $\mathrm{Pa}$, USA, 2017.

[60] D. Minoli, K. Sohraby, and J. Kouns, "IoT Security (IoTSec) Considerations, Requirements," in Proceedings of the 14th IEEE Annual Consumer Communications \& Networking Conference (CCNC), IEEE, Las Vegas, NV, USA, 2017.

[61] "Security architecture and procedures for 5G System," 3GPP TS 33.501, 2018, http://www.3gpp.org/DynaReport/33501.htm.

[62] "Study on the security aspects of the next generation system," 3GPP TR 33.899, 2017, http://www.3gpp.org/DynaReport/ 33899.htm.

[63] "5G Enablers for Network and System Security and Resilience," http://www.5gensure.eu/, 2017.

[64] GDPR, General Data Protection Regulation, European Union, 2016, http://eur-lex.europa.eu/legal-content/en/ALL/?uri= CELEX:32016R0679.

[65] ePrivacy, Directive on privacy and electronic communications, European Union, 2002, https://eur-lex.europa.eu/legalcontent/en/ALL/?uri=CELEX:32002L0058.

[66] The Strait Times Staff, China to Be Biggest 5G Market by 2025: Report, 2019, https://www.straitstimes.com/world/ china-to-be-biggest-5g-market-by-2025-report.

[67] J. Stubbs and D. Busvine, Weak investment climate main 5 G risk, not security fears: Ericsson, https://www.reuters.com/article/ us-telecoms-mobileworld-ericsson/weak-investment-climatemain-5g-risk-not-security-fears-ericsson-idUSKCN1QE0ST.

[68] FCC, Millimeter Wave Propagation: Spectrum Management Implications, vol. 70, Federal Communications Commission Office of Engineering and Technology New Technology Development Division, Washington, DC, USA, 1997.
[69] P. Tracy, What is Mm Wave and How Does It Fit into 5G?, 2016, https://www.rcrwireless.com/20160815/fundamentals/ mmwave-5g-tag31-tag99.

[70] X. Zhang, Millimeter Wave for 5G: Unifying Communication and Sensing, 2015, https://www.microsoft.com/en-us/research/wpcontent/uploads/2015/03/Xinyu-Zhang_5GmmWave.pdf.

[71] X. Ge, J. Yang, H. Gharavi, and Y. Sun, "Energy efficiency challenges of 5G small cell networks," IEEE Communications Magazine, vol. 55, no. 5, pp. 184-191, 2017.

[72] X. Ge, Z. Li, and S. Li, "5G software defined vehicular networks," IEEE Communications Magazine, vol. 55, no. 7, pp. 87-93, 2017.

[73] S. Sun, T. S. Rappaport, S. Rangan et al., "Propagation path loss models for 5G urban micro- and macro-cellular scenarios," in Proceedings of the 83rd IEEE Vehicular Technology Conference (VTC Spring), IEEE, Nanjing, China, 2016.

[74] "Draft declaratory ruling and third report and order," WC Docket No. 17-84, WT Docket No.17-79, FCC-CIRC1809-02, 2018.

[75] "Small cell forum," Small Cells Market Status Report, 2018, http://www.scf.io/en/documents/050_Small_cells_market_status_report_February_2018.php?utm_source=Email\%campaign \& utm_medium=eshots\&utm_campaign=member\%eshot.

[76] T. S. Rappaport, Y. Xing, G. R. MacCartney et al., "Overview of millimeter wave communications for fifth-generation $(5 \mathrm{G})$ wireless networks-with a focus on propagation models," IEEE Transactions on Antennas and Propagation, vol. 65, no. 12, pp. 6213-6230, 2017.

[77] T. Rappaport, S. Sun, R. Mayzus et al., "Millimeter wave mobile communications for $5 \mathrm{G}$ cellular," IEEE Access, vol. 1, pp. 335349, 2013.

[78] T. Bai, A. Alkhateeb, and R. W. Heath, "Coverage and capacity of millimeter-wave cellular networks," IEEE Communications Magazine, vol. 52, no. 9, pp. 70-77, 2014.

[79] S. Rangan, T. S. Rappaport, and E. Erkip, "Millimeter-wave cellular wireless networks: potentials and challenges," Proceedings of the IEEE, vol. 102, no. 3, pp. 366-385, 2014.

[80] W. Roh, J.-Y. Seol, J. Park et al., "Millimeter-wave beamforming as an enabling technology for $5 \mathrm{G}$ cellular communications: theoretical feasibility and prototype results," IEEE Communications Magazine, vol. 52, no. 2, pp. 106-113, 2014.

[81] 3GPP 38.901-e20, “3rd generation partnership project," Technical Specification Group Radio Access Network; Study on Channel Model For Frequencies From 0.5 to $100 \mathrm{GHz}$ (Release 14), 2017.

[82] F. Khan and Z. Pi, "mmWave mobile broadband (MMB): unleashing the 3-300GHz spectrum," in Proceedings of the 34th IEEE Sarnoff Symposium, pp. 1-6, Princeton, NJ, USA, 2011.

[83] Z. Pi and F. Khan, "An introduction to millimeter-wave mobile broadband systems," IEEE Communications Magazine, vol. 49, no. 6, pp. 101-107, 2011.

[84] F. Khan and Z. Pi, "Millimeter-wave mobile broadband: unleashing 3-300 GHz spectrum," in Proceedings of the IEEE Wireless Communications and Networking Conference, IEEE, 2011.

[85] S. Rajagopal, S. Abu-Surra, Z. Pi, and F. Khan, "Antenna array design for multi-Gbps mmwave mobile broadband communication," in Proceedings of the IEEE Global Telecommunications Conference (GLOBECOM), pp. 1-6, Houston, Tex, USA, 2011.

[86] A. Ghosh, T. A. Thomas, M. C. Cudak et al., "Millimeterwave enhanced local area systems: a high-data-rate approach for future wireless networks," IEEE Journal on Selected Areas in Communications, vol. 32, no. 6, pp. 1152-1163, 2014. 
[87] G. R. MacCartney and T. S. Rappaport, "Study on 3GPP rural macrocell path loss models for millimeter wave wireless communications," in Proceedings of the ICC 2017 - 2017 IEEE International Conference on Communications, pp. 1-7, IEEE, Paris, France, 2017.

[88] Y.-S. Lu, C.-F. Lai, C.-C. Hu, and Y.-M. Huang, "Path loss exponent estimation for indoor wireless sensor positioning," KSII Transactions on Internet and Information Systems, vol. 4, no. 3, article 243, 2010.

[89] S. Srinivasan and M. Haenggi, "Path loss exponent estimation in large wireless networks," Information Theory and Applications Workshop, pp. 124-129, 2009.

[90] M. Viswanathan, Log Distance Path Loss or Log Normal Shadowing Model, 2013, https://www.gaussianwaves.com/2013/09/ log-distance-path-loss-or-log-normal-shadowing-model/.

[91] G. L. Lederer, "Smart communities and special districts coalition - ex parte submission: accelerating wireless broadband deployment by removing barriers to infrastructure investment WT docket no. 17-79 and no. 17-84," Best, Best \& Krieger, LLP, 2018, 2000 Pennsylvania Avenue, N.W., Suite 5300, Washington, DC 20006.

[92] GOS World Staff, Skyworks Unveils Sky5 Ultra Platform for 5G Architecture, 2019, https://www.gpsworld.com/skyworksunveils-sky5-ultra-platform-for-5g-architecture/.

[93] J. Sanders, Tracking 5G: Ookla’s Coverage Map Tracks Worldwide Network Rollout, 2019, https://www.techrepublic.com.

[94] C. Yorkgitis, FCC Adopts a Second Wave of Millimeter Wave Regulations to Support Next Generation Terrestrial Systems and Services, Common Law Monitor, 2017, https://www.commlawmonitor.com/2017/12/articles/wireless-2/fcc-adopts-a-secondwave-of-millimeter-wave-regulations-to-support-next-generation-terrestrial-systems-and-services/.

[95] Y. Saleem, N. Crespi, M. H. Rehmani, and R. Copeland, "Internet of things-aided smart grid: technologies, architectures, applications, prototypes, and future research directions," IEEE Access, vol. 7, pp. 62962-63003, 2019.

[96] Y. Li, X. Cheng, Y. Cao, D. Wang, and L. Yang, "Smart choice for the smart grid: narrowband internet of things (NB-IoT)," IEEE Internet of Things Journal, vol. 5, no. 3, pp. 1505-1515, 2018.

[97] P. Reininger, "3GPP standards for the internet of-things," https://www.slideshare.net/eikoseidel/3gpp-standards-for-theinternetofthings, 11 3gpp Standards for IoT.pdf, 2016.

[98] "cellular system support for ultra-low complexity and low throughput internet of things (CIoT)," https://portal.3gpp.org/ desktopmodules/Specifications/SpecificationDetails.aspx?specificationId $=2719$.

[99] R. Ratasuk, B. Vejlgaard, N. Mangalvedhe, and A. Ghosh, "NB-IoT system for M2M communication," in Proceedings of the IEEE Wireless Communications and Networking Conference (WCNC), pp. 1-5, 2016.

[100] Link Labs Staff, An Overview of Narrowband IoT (NB-IoT), 2018, https://www.link-labs.com/blog/overview-of-narrowband-iot.

[101] Y. E. Wang, X. Lin, A. Adhikary et al., "A primer on 3GPP narrowband internet of things," IEEE Communications Magazine, vol. 55, no. 3, pp. 117-123, 2017.

[102] “T-Mobile NB-IoT plan," https://iot.t-mobile.com/pricing/.

[103] "Verizon wireless Cat-M Plan," https://www.verizonwireless .com/biz/plans/m2m-business-plans/.

[104] GSMA, Mobile IoT in the 5G Future- NB-IoT and LTE-M in the context of 5G, 2018, https://www.gsma.com/iot/wpcontent/uploads/2018/05/GSMAIoT_MobileIoT_5G_Future_ May2018.pdf.
[105] M. Contento, $5 G$ and IoT - Emerging Tech with Endless Use Cases, 2019, https://www.telit.com/blog/state-of-5g-and-iotcurrent-future-applications/.

[106] The Guardian View on Google Versus Huawei: No Winners, The Guardian, 2019, https://www.theguardian.com/commentisfree/ 2019/may/20/the-guardian-view-on-google-versus-huawei-nowinners. 


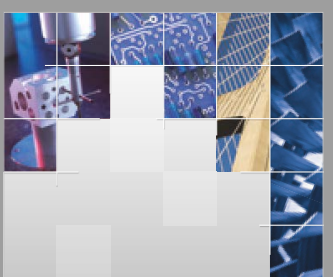

\section{Enfincering}
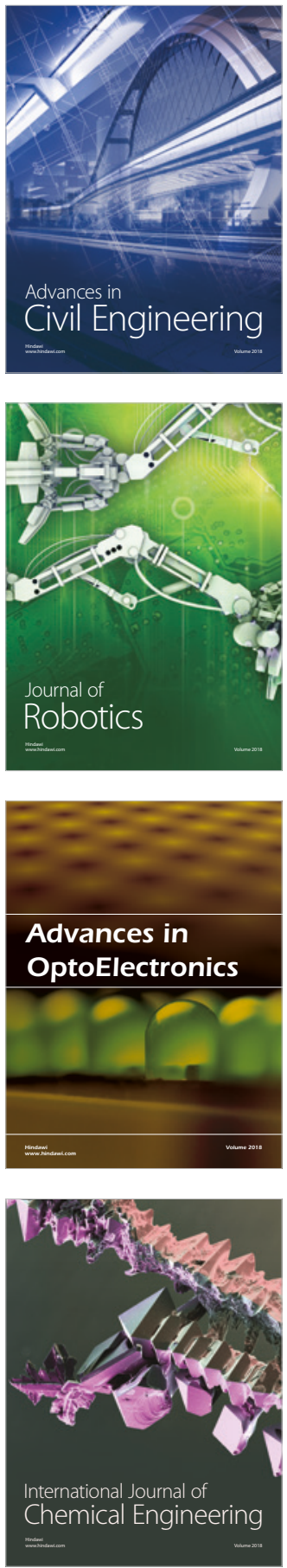

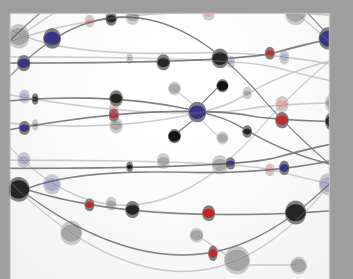

\section{Rotating \\ Machinery}

The Scientific World Journal

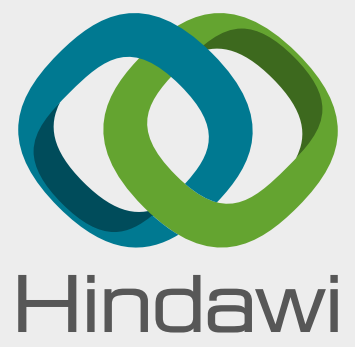

Submit your manuscripts at

www.hindawi.com
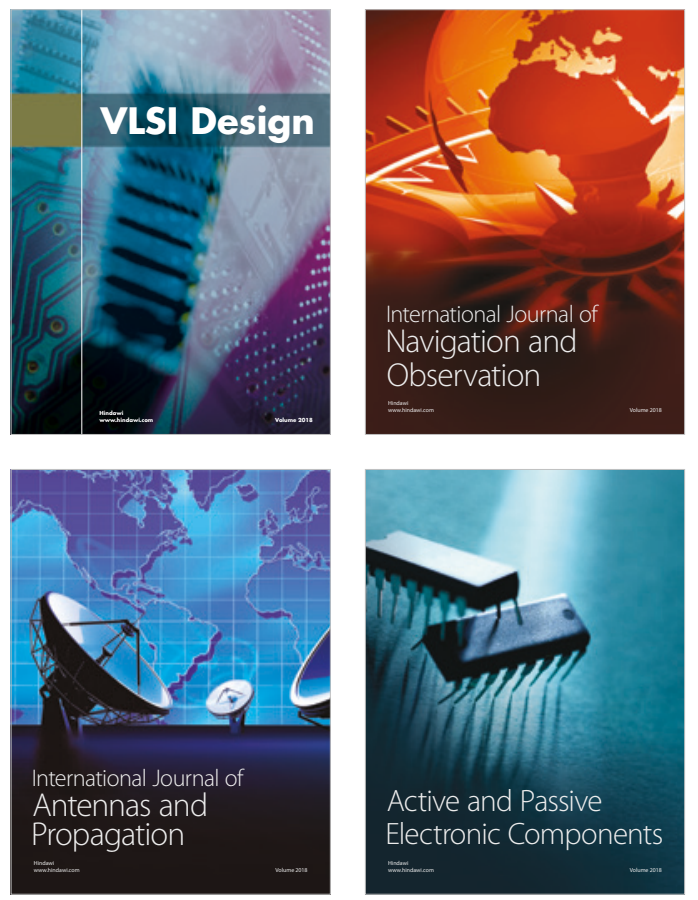
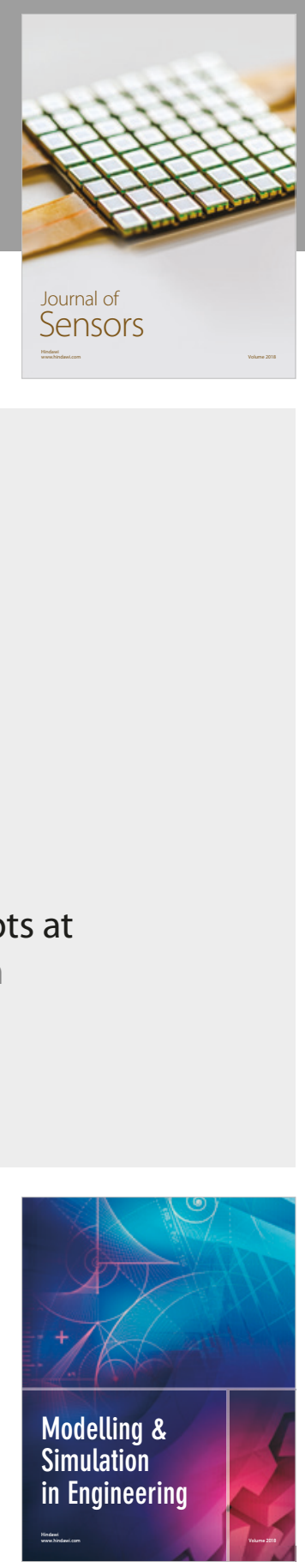

\section{Advances \\ Multimedia}
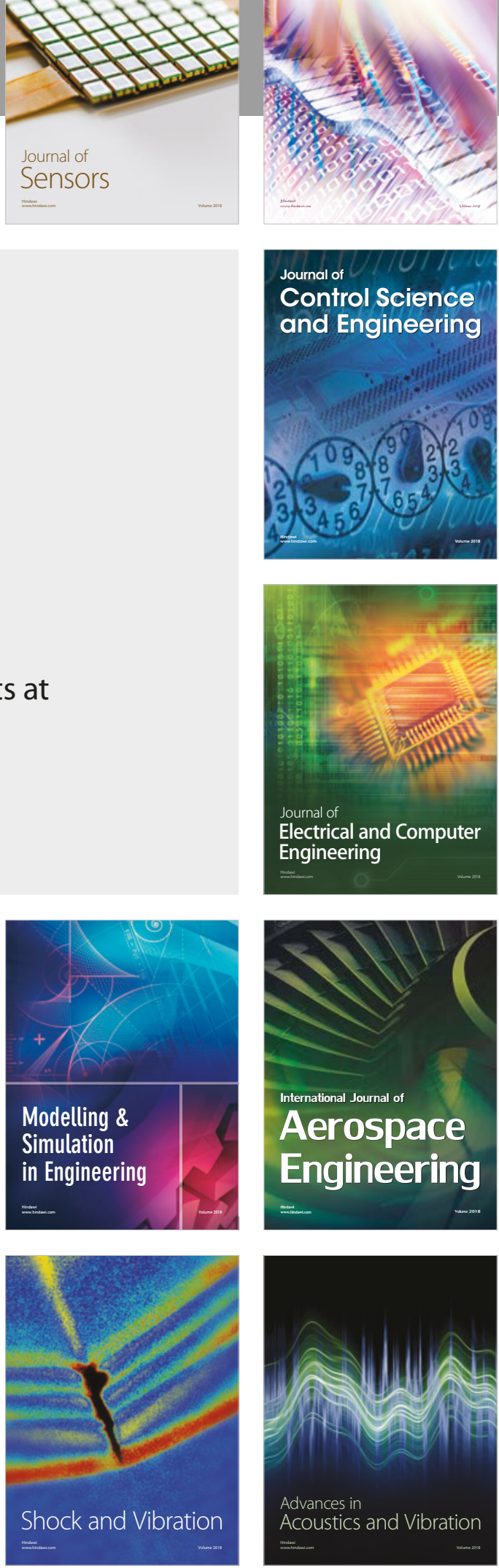